\title{
Identification of diagnostic genes and vital microRNAs involved in rheumatoid arthritis: based on data mining and experimental verification
}

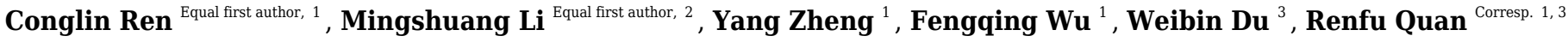 \\ ${ }^{1}$ The Third Clinical Medical College of Zhejiang Chinese Medical University, Hangzhou, Zhejiang, China \\ 2 The First Affiliated Hospital of Zhejiang Chinese Medical University, Hangzhou, Zhejiang, China \\ 3 Department of Orthopedics, Xiaoshan Traditional Chinese Medicine Hospital, Hangzhou, Zhejiang, China \\ Corresponding Author: Renfu Quan \\ Email address: quanrf@yeah.net
}

Background. The pathogenesis of rheumatoid arthritis (RA) is complex. This study aimed to identify diagnostic biomarkers and transcriptional regulators that underlie RA based on bioinformatics analysis and experimental verification. Material and Methods. We applied weighted gene co-expression network analysis (WGCNA) to analyze dataset GSE55457 and obtained the key module most relevant to the RA phenotype. We then conducted gene function annotation, gene set enrichment analysis (GSEA) and immunocytes quantitative analysis (CIBERSORT). Moreover, the intersection of differentially expressed genes (DEGs) and genes within the key module were entered into the STRING database to construct an interaction network and to mine hub genes. We predicted microRNA (miRNA) using a webbased tool (miRDB). Finally, hub genes and vital miRNAs were validated with independent GEO datasets, RT-qPCR and Western blot. Results. A total of 367 DEGs were characterized by differential expression analysis. The WGCNA method divided genes into 14 modules, and we focused on the turquoise module containing 845 genes. Gene function annotation and GSEA suggested that immune response and inflammatory signaling pathways are the molecular mechanisms behind RA. Nine hub genes were screened from the network and seven vital regulators were obtained using miRNA prediction. CIBERSORT analysis identified five cell types enriched in RA samples, which were closely related to the expression of hub genes. Through ROC curve and RT-qPCR validation, we confirmed five genes that were specific for RA, including CCL25, CXCL9, CXCL10, CXCL11, and CXCL13. Moreover, we selected a representative gene (CXCL10) for Western blot validation. Vital miRNAs verification showed that only the differences in has-miR-573 and has-miR-34a were statistically significant. Conclusion. Our study reveals diagnostic genes and vital microRNAs highly related to RA, which could help improve our understanding of the molecular mechanisms underlying the disorder and provide theoretical support for the 
future exploration of innovative therapeutic approaches. 
1 Identification of diagnostic genes and vital microRNAs involved in rheumatoid arthritis based on data mining and experimental verification

3

4 Conglin Ren ${ }^{1 *}$, Mingshuang $\mathrm{Li}^{2 *}$, Yang Zheng ${ }^{1}$, Fengqing $\mathrm{Wu}^{1}$, Weibin $\mathrm{Du}^{3}$, and Renfu Quan ${ }^{1,3}$

5 1The Third Clinical Medical College of Zhejiang Chinese Medical University, Hangzhou,

6 Zhejiang 310051, China

$7{ }^{2}$ The First Affiliated Hospital of Zhejiang Chinese Medical University, Hangzhou, Zhejiang

$8 \quad 310002$, China

$9{ }^{3}$ Department of Orthopedics, Xiaoshan Traditional Chinese Medicine Hospital, Hangzhou, 10 Zhejiang 311200, China

12 Corresponding Author: Renfu Quan.

13 YucaiRoad 156, XiaoshanDist, Hangzhou, Zhejiang 311200, China

14 Email address: quanrf@yeah.net

*These authors contributed equally to this work. 
Abstract

Background. The pathogenesis of rheumatoid arthritis (RA) is complex. This study aimed to identify diagnostic biomarkers and transcriptional regulators that underlie RA based on bioinformatics analysis and experimental verification.

Material and Methods. We applied weighted gene co-expression network analysis (WGCNA) to analyze dataset GSE55457 and obtained the key module most relevant to the RA phenotype. We then conducted gene function annotation, gene set enrichment analysis (GSEA) and immunocytes quantitative analysis (CIBERSORT). Moreover, the intersection of differentially expressed genes (DEGs) and genes within the key module were entered into the STRING database to construct an interaction network and to mine hub genes. We predicted microRNA (miRNA) using a web-based tool (miRDB). Finally, hub genes and vital miRNAs were validated with independent GEO datasets, RT-qPCR and Western blot.

Results. A total of 367 DEGs were characterized by differential expression analysis. The WGCNA method divided genes into 14 modules, and we focused on the turquoise module containing 845 genes. Gene function annotation and GSEA suggested that immune response and inflammatory signaling pathways are the molecular mechanisms behind RA. Nine hub genes were screened from the network and seven vital regulators were obtained using miRNA prediction. CIBERSORT analysis identified five cell types enriched in RA samples, which were closely related to the expression of hub genes. Through ROC curve and RT-qPCR validation, we confirmed five genes that were specific for RA, including CCL25, CXCL9, CXCL10, CXCL11, and CXCL13. Moreover, we selected a representative gene (CXCL10) for Western blot validation. Vital miRNAs verification showed that only the differences in has-miR-573 and has-miR-34a were statistically significant.

Conclusion. Our study reveals diagnostic genes and vital microRNAs highly related to RA, which could help improve our understanding of the molecular mechanisms underlying the disorder and provide theoretical support for the future exploration of innovative therapeutic approaches. 


\section{Introduction}

Rheumatoid arthritis (RA) is a disorder characterized by symmetrical, aggressive joint inflammation(Smolen et al. 2016). The prevalence of RA varies somewhat by ethnicity and geographic region. According to the literature, African Americans have a higher prevalence of the disease by approximately $1.02 \%$, compared to Hispanic Americans, who have a much lower risk of this disease at only $0.45 \%($ Kawatkar et al. 2012). The etiology of RA is unclear, and current research suggests that it may result from a combination of factors, including genetics, infection, and sex hormones. A study on twins showed that the genetic probability of RA is approximately $60 \%$ and is not influenced by sex, age of onset, or disease severity(MacGregor et al. 2000). The changes in the composition and function of gut microbes are closely related to infection by autoimmune diseases. The microbial diversity of the gut is significantly reduced in RA patients compared to healthy individuals, and the proportion of certain rare bacteria like Actinobacteria is elevated(Chen et al. 2016). More interestingly, oral infections whose clinical manifestations seem unrelated to RA have also been identified as risk factors in recent studies(Hajishengallis 2015; Kharlamova et al. 2016). The pathogenesis of RA is complex and clinical symptoms vary considerably between individuals. There have been no reliable biomarkers identified to date, despite the discovery of multiple susceptibility genes associated with RA (e.g., IL2RA and MMP9) and new advances in the development of therapeutic drugs.

microRNA (miRNA) has become a key node in biomedical research as an important factor regulating gene transcription and post-transcriptional regulation. Numerous findings support the idea that miRNAs are essential for maintaining immune system homeostasis, including the regulation of T-cell activation, immune tolerance and the inflammatory response, and the aberrant expression of miRNAs may lead to increased susceptibility to autoimmune diseases(O'connell et al. 2010a; Simpson \& Ansel 2015). Recently, the deregulation of endogenous miR-155 has been shown to be involved in malignant tumorigenesis and in abnormal autoimmune conditions such as RA and systemic sclerosis(Leng et al. 2011). Using miRNA profiling, Pan et al. (2010) found that miR-21 and miR-148a were highly expressed in T cells from lupus patients and animal models, 
which inhibited DNMT1 gene expression to induce cellular hypomethylation(Pan et al. 2010). Therefore, an in-depth study of the functional links between miRNAs and mRNAs may reveal the pathogenesis of diseases and develop new therapeutic approaches.

With the advent of microarray and high-throughput sequencing technologies, bioinformatics is becoming a popular discipline in biomedical research, and analysis tools are constantly being developed and upgraded. Weighted gene co-expression network analysis (WGCNA) is an algorithm for detecting correlation between genes(Langfelder \& Horvath 2008; Langfelder \& Horvath 2012), which facilitates network-based genetic screening methods and is helpful to uncover potential biomarkers and therapeutic targets for diseases. WGCNA has been successfully practiced in studies of autoimmune diseases, such as juvenile dermatomyositis(Zhong et al. 2020), lupus nephritis(Yang \& Li 2019), ulcerative colitis(Zhang et al. 2020) and Sjögren's syndrome(Yao et al. 2019). We integrated multiple bioinformatics methods to analyze microarray datasets derived from the Gene Expression Omnibus (GEO) repository and validated the data mining results in three ways in order to identify diagnostic genes and transcriptional regulators of RA. Figure 1 summarized the entire process of our research.

\section{Material and Methods}

\section{Data download and processing}

Gene expression matrices and the clinical information of GSE55457 based on platform GPL96 [HG-U133A], were downloaded from the GEO database. Twenty-three samples were selected for our study. We performed normalization and $\log 2$ transformation on original expression matrices and used a platform annotation file to convert probes into gene symbols. If a gene was detected by more than one probe, the average expression value was used for subsequent analysis.

Differentially expressed genes (DEGs) between RA patients and healthy controls were analyzed by limma, which is a tailored R package for expression profiling studies(Ritchie et al. 2015). The DEGs filtering thresholds were set to $|\log F C|>1$ and the adjusted p-value was $<0.05$.

\section{Weighted correlation network analysis}

Gene co-expression analysis of dataset GSE55457 was performed in R software (version: 3.6.3) 
103

104

105

106

107

108

109

110

111

112

113

114

115

116

117

118

119

120

121

122

123

124

125

126

127

128

129

based on the WGCNA R package reference manual. We calculated the median absolute deviation (MAD) for each gene, which reflected the degree of variation in gene expression across samples, and selected the top 3,000 highly variable genes for network construction and modules detection. First, we clustered samples to detect the presence of outliers. Next, optimal soft-thresholding power $(\beta)$ was selected, which was a pivotal step in the entire analysis process. In order to reduce interference from spurious correlations, the adjacency matrix was replaced by a topological overlap matrix (TOM), and then the hclust function was used to generate a hierarchical clustering tree (dendrogram) of genes. The detailed parameters used for modules detection were as follows: minModuleSize was set to 30 to obtain large modules; modules with a correlation of 0.75 were merged using mergeCloseModules function. We also quantified module-trait associations and their p-values, which allowed us to pick out modules that were closely related to the trait of interest for next step analysis. The absolute value of correlation coefficient $>0.6$ and $p$-value $<0.05$ were cutoffs for module screening. Finally, we measured the gene significance (GS) and module membership (MM) of individual genes and scatterplots were drawn.

\section{Functional annotation and GSEA}

R package clusterProfiler(Yu et al. 2012) and its dependency annotation package org.Hs.eg.db, sourced from Bioconductor, were used to analyze and visualize the functional profiles (GO and KEGG) of key module genes. To obtain highly credible analysis results, only enrichment items satisfying both $\mathrm{p}$-value $<0.05$ and q-value $<0.05$ were outputted. Moreover, gene set enrichment analysis (GSEA)(Mootha et al. 2003; Subramanian et al. 2005), which can be used to determine whether an already-defined gene set exhibits a statistically significant difference in two different traits (e.g., RA patients and healthy individuals), was conducted to explore biological processes and pathways that may be involved in the pathogenesis of RA. The complete gene expression matrix and phenotype information of dataset GSE55457 were uploaded to GSEA and analyzed with hallmark gene sets that are stored in molecular signatures database (MSigDB)(Liberzon et al. 2015; Liberzon et al. 2011). Gene sets enriched at FDR $<0.25$ and p-value $<0.01$ were retained.

\section{Building PPI network and mining hub genes}


130 The intersection of DEGs and key module genes was entered into a STRING database to find 131 interrelationships(Szklarczyk et al. 2015; Szklarczyk et al. 2019; Szklarczyk et al. 2017). Due to 132 the large size of the initial protein-protein interaction (PPI) network, we hid isolated nodes and 133 limited the minimum interaction score to 0.7 . Then, the network was exported and further

134

135

136

137

138

139

140

141

142

143

144

145

146

147

148

149

150

151

152

153

154

155

156 optimized in Cytoscape software(Cline et al. 2007; Otasek et al. 2019; Shannon et al. 2003). The MCODE plugin was installed in Cytoscape to find clusters made up of highly related genes in the network(Bader \& Hogue 2003). The parameters used in our analysis were default values. Genes in the highest scoring cluster were considered to be hub genes of RA and were re-performed for KEGG analysis to explore vital biological pathways.

Estimating proportion of immune cells in synovial tissue

Understanding the immune microenvironment in RA may provide new insights into the immunotherapy of the disease. The CIBERSORT tool used for estimating the immune cell contents of different samples in a gene expression admixture has a wide range of applicability and high sensitivity, which is important for disease diagnosis and therapeutic target predictions(Newman et al. 2015). A mixture file was generated according to the input format requirements, and we selected default LM22 as signature gene file to distinguish the hematopoietic populations and activation states. The analysis results were filtered based on a threshold of p-value $<0.05$ to remove samples with a low confidence level. We conducted correlation analysis between the hub genes and immune cell subtypes significantly enriched in RA samples according to Pearson's algorithm.

\section{Prediction of gene-miRNA relationships}

MiRNAs are a class of non-coding RNAs that regulate gene expression by making mRNA unstable and inhibiting translation, a process by which mRNA is converted into protein. Here, MCODEidentified hub genes were uploaded to the online analysis tool miRDB; we searched for miRNAs that have regulatory roles in the development of RA(Chen \& Wang 2020; Liu \& Wang 2019). The parameters were set as follows: only functional miRNAs were considered; gene targets with a score below 60 and miRNAs with more than 2,000 targets were excluded. Cytoscape was then 
157

158

159

160

161

162

163

164

165

166

167

168

169

170

171

172

173

174

175

176

177

178

179

180

181

182

183

used to construct a gene-miRNA network in which miRNAs targeting two or more genes were considered to be vital regulators involved in RA pathogenesis.

\section{Validating hub genes and miRNAs with independent microarray datasets}

The expression matrix GSE12021 was selected as the validation dataset for hub genes. We plotted ROC curves in R software based on the pROC package to test whether screened hub genes have good diagnostic value for RA(Robin et al. 2011). The area under the curve (AUC) was computed according to the trapezoidal rule and genes with AUC $>85 \%$ were further validated by RT-qPCR. To understand the differences in transcriptional regulators between RA patients and healthy individuals, we validated vital miRNAs with GEO dataset GSE37425, which focused on the expression of miRNAs in synovial tissue using the TaqMan probe approach. The entire gene expression matrix was normalized and then transformed with $\log 2$.

\section{Validating hub genes and miRNAs with RT-qPCR}

Five RA patients and three healthy individuals who attended the health examination center of Xiaoshan Traditional Chinese Medicine Hospital were included and all subjects signed consent forms. This study was approved by Ethics Committees of Xiaoshan Traditional Chinese Medicine Hospital (NO. 2020012). We collected blood samples from each test subject and isolated the peripheral blood mononuclear cells (PBMCs) using human lymphocyte separation medium (Solarbio, China). Next, the total RNA of PBMCs was extracted using the TRIzol reagent (Invitrogen, USA) and its purity was assessed by NanoDrop (Thermo Scientific, USA). We used a cDNA synthesis kit (Thermo Scientific, USA) to generate the first strand cDNA and cDNA amplification was performed on a 7500 real-time PCR instrument (Applied Biosystems, USA). For the quantitative analysis of miRNA expression, Hairpin-it miRNAs qPCR quantitation kit (Genepharma, China) was applied. We chose GAPDH and U6 as internal reference genes and compared relative mRNA or miRNA expression based on the $2^{-\Delta \Delta C T}$ method. The primer sequences are available in Table 1.

\section{Western blot}

Total proteins were extracted from PBMCs using RIPA lysis buffer (Beyotime Biotechnology, 
184

185

186

187

188

189

190

191

192

193

194

195

196

197

198

199

200

201

202

203

204

205

206

207

208

209

210

China), followed by concentration detection with the BCA protein assay kit (Sangon Biotech, China). Equal amounts of protein were separated by $8-20 \%$ SDS-PAGE and transferred onto polyvinylidene difluoride membranes. Afterwards, membranes were blocked with 5\% skim milk

for 1 hour and then incubated with primary antibodies including $\beta$-actin $(1: 1000$, Cell Signaling Technology (CST),\#3700), and CXCL10 (1:1000, CST, \#14969) at $4^{\circ} \mathrm{C}$ overnight. Anti-mouse $\operatorname{IgG}(1: 2000$, CST, \#7076) and anti-rabbit $\operatorname{IgG}(1: 2000$, CST, \#7074) were used to bind the primary antibodies. Finally, membranes were scanned using FluorChem Q (Proteinsimple, USA). Image J software was used for the quantitative analysis of protein bands.

\section{Statistical analysis}

Statistical analysis was performed in GraphPad Prism 7.0. Data satisfying normal distribution were analyzed by unpaired t-test; the Mann-Whitney test was used for data that were not normalized. P-value $<0.05$ was considered significant.

\section{Results}

\section{Screening for DEGS}

Our differential expression assessment of dataset GSE55457 identified 367 DEGs, of which 186 genes were highly expressed and 181 genes had low expression levels in RA patients. We created a volcano map and heat map for DEGs as shown in Figure 2.

\section{Identification of key module closely related to $R \boldsymbol{A}$}

Two samples (GSM1337307 and GSM1337308) were identified as outliers and were excluded by the cutreeStatic function with parameter cutHeight $=90$ (Fig 3A). The soft-thresholding power $(\beta)$ was set to 5, for which the scale-free topology fit index $\left(\mathrm{R}^{2}\right)$ reached 0.90 (Fig 3B). Co-expression analysis resulted in 14 modules, and the size of each module is shown in Figure 3C and 3D. Figure 3E showed the relationships between modules and traits, with four modules (black, turquoise, blue, and purple) having strong positive or negative correlations with disease status (presence or absence of RA). Taking into account gene numbers, correlation coefficients, and p-values of the above four modules, we focused on the turquoise module containing 845 genes and performed an in-depth analysis. The GS and MM plot of the turquoise module (correlation coefficient $=0.79$, p-value $=$ 
$2112.7 \mathrm{e}^{-181}$ ) demonstrated that the closer the gene is to the trait, the more important it is in the module $212($ Fig 3F).

\section{Functional annotation of turquoise module genes and GSEA}

214 The top 10 results for GO enrichment are shown in Figure 4A and mainly involved leukocyte 215 activation and cell adhesion. To associate a specific gene with its assigned GO terms, we adopted 216 the GOChord function to generate a circle diagram (Fig 4B). Figure 4C pointed out that 217 inflammation-related signaling pathways, such as chemokine signaling pathway, NF-kappa B 218 signaling pathway, Th17 cell differentiation and T cell receptor signaling pathway, were 219 significantly linked to the pathogenesis of RA. Similarly, a circle plot was displayed to visualize 220 the correspondence between genes and pathways (Fig 4D). We identified five gene sets based on 221 a reference database derived from MSigDB using the GSEA method. These were associated with 222 the immune response or inflammatory pathways that were highly enriched in RA, which verified 223 the GO and KEGG results (Fig 5A-E).

224

225

226

227

228

229

230

231

232

233

234

235

236

237

\section{Constructing PPI network to find hub genes}

A total of 171 intersection genes were entered into the STRING database to establish the network (Fig 6A). The network was adjusted using Cytoscape software, resulting in 52 nodes and 169 relationship pairs. The MCODE plugin uncovered five clusters, with the highest scoring cluster containing nine nodes, which were potential hub genes for RA (Fig 6B, Table 2). Furthermore, we re-performed KEGG analysis on the nine genes to investigate the signaling pathways in which they were involved. As shown in Figure 6C, we found that nine hub genes played important roles in inflammatory or immune-related pathways, including the chemokine signaling pathway, tolllike receptor signaling pathway, and TNF signaling pathway.

\section{Immune infiltration and Pearson correlation analysis}

One sample (GSM1337307) was excluded from analysis results because its p-value did not meet the threshold we set. The contents of various immune cells in each sample were presented by stacked bar chart (Fig 7A). M2 macrophages accounted for the highest proportion in both RA and normal tissue. We conducted a Wilcoxon test to further clarify whether the level of each immune 
238

239

240

241

242

243

244

245

246

247

248

249

250

251

252

253

254

255

256

257

258

259

260

261

262

263

264

cell type was statistically different between two groups. From the violin plot (Fig 7B), we inferred that five cell types, including plasma cells, CD8 T cells, follicular helper T cells, $\gamma \delta \mathrm{T}$ cells, and M1 macrophages, were abundant in RA synovial tissue compared to the normal group. More importantly, Pearson correlation analysis confirmed that, in addition to CCL25, eight other hub genes identified by MCODE played pro-inflammatory roles. Their overexpression may recruit large numbers of inflammatory cells to lesion sites, which is a critical pathological basis for RA (Fig 7C).

\section{Predicting miRNAs that regulate nine hub genes}

Normally, miRNAs can fine-tune gene expression to maintain the homeostasis of life, while abnormal miRNAs expression is thought to be associated with diseases, including benign or malignant tumors, and immune system dysfunction. Our prediction of miRNAs was made in the miRDB database and 72 miRNA-gene regulatory relationships were obtained. We then created an interactive network in Cytoscape and analyzed the number of genes targeted by each miRNA (Fig 8). The miRNAs possessing two target genes are listed in Table 3 and were vital regulators in the pathogenesis of RA.

\section{Validation of hub genes by ROC curves}

The expression matrix of dataset GSE12021 was chosen to test the diagnostic efficacy of hub biomarkers for RA. Probability curves (ROC curves) were plotted and the AUC reflected the degree or measure of separability. A higher AUC indicated that the gene is better at discriminating between diseased and non-diseased individuals. As shown in Figure 9, six genes had AUC values greater than 85\%, including CCL25, CXCL10, CCL5, CXCL9, CXCL13, and CXCL11, indicating that these genes were potential biomarkers for the diagnosis of RA.

\section{Validation of hub genes by RT-qPCR and Western blot}

We collected peripheral blood from subjects for RT-qPCR validation to further evaluate the six genes with AUC $>85 \%$ identified by ROC curves. Five genes were differentially expressed between RA patients and normal subjects (Fig 10A). Among them, CXCL9, CXCL10, CXCL11, and CXCL13 were up-regulated genes, while CCL25 was down-regulated. Moreover, to detect the 
265

266

267

268

269

270

271

272

273

274

275

276

277

278

279

280

281

282

283

284

285

286

287

288

289

290

291

expression of genes in protein level, we chose a representative gene (CXCL10) for Western blot validation. As shown in Figure 10B and C, the protein and mRNA level of gene expression were consistent.

\section{Vital miRNAs verification}

The vital miRNAs possessing two target genes were validated with GSE37425. Among the six miRNAs investigated, has-miR-449b, has-miR-573, and has-miR-203 expression was suppressed in RA patients (Fig 11A-F). It is worth noting that only the differences in has-miR-573 and hasmiR-34a were statistically significant. Previous studies have reported that miR-34a played an important role in the pathogenesis of RA, but the findings were controversial(Niederer et al. 2012; Xie et al. 2019). Hence, we analyzed the expression of miR-34a-5p in PBMCs using RT-qPCR and found that miR-34a-5p was significantly down-regulated in RA patients compared to health controls (Fig 11G).

\section{Discussion}

We integrated multiple bioinformatics analysis methods to attain diagnostic genes and vital microRNAs of RA. A total of 14 modules were identified using the WGCNA algorithm. Of these, the turquoise module was large in size (containing 845 genes) and highly correlated with RA, and was selected for further study. We found that the main functions of genes were to participate in leukocyte activation and regulate cell-cell adhesion by performing $\mathrm{GO}$ annotation on the turquoise module. KEGG results showed that these genes were highly focused on cytokine-cytokine receptor interaction, chemokine signaling pathway, NF-kappa B signaling pathway, and T cell receptor signaling pathway, in addition to regulating helper $\mathrm{T}(\mathrm{Th})$ cell differentiation. Cytokines are small peptides or glycoproteins produced by stimulated immune cells and some stromal cells (e.g., endothelial cells) with a wide range of biological activities(Lackie 2010). They can mediate longlasting tissue inflammation and injury by autocrine or paracrine systems and have been shown to be implicated in the pathogenesis of many host autoimmune diseases, such as RA, inflammatory bowel disease, and ankylosing spondylitis(Schett et al. 2013). It is believed that TNF and IL-6 are critical pro-inflammatory cytokines that promote osteoclast maturation leading to cartilage 
292 degeneration and matrix degradation, and can induce the release of other stimulating factors (such 293 as IL-1), resulting in a vicious cycle of unrelieved synovitis in RA(Bertolini et al. 1986; Ohshima 294 et al. 1998). The NF-kappa B signaling pathway, activated by the receptor activator of nuclear 295 factor $\kappa \mathrm{B}$ ligand (RANKL), is also worthy of attention for its pathogenic effect on RA. Hirayama 296 et al (2005). found that blocking the NF-kappa B pathway with STAT-6 fusion protein 297 significantly alleviated joint inflammation and bone destruction in mice(Hirayama et al. 2005). 298 Moreover, GSEA analysis identified five inflammation-associated gene sets that were upregulated in the RA phenotype, such as IL6-JAK-STAT3 signaling, interferon $\alpha / \gamma$ response and complement, which were highly consistent with the results of GO and KEGG annotation. The Janus kinase (JAK) family, consisting of four JAK proteins, transduces inflammatory cytokines signals (e.g., IL-6 and interferon) through the JAK-STAT pathway. A double-blind clinical study showed promising clinical improvement in RA patients after oral administration of tofacitinib, a drug that blocks the JAK1-mediated cytokine signaling and inhibits STAT phosphorylation(Boyle et al. 2015). We used CIBERSORT to quantify the composition and content of immune cells in synovial tissue to further understand the immune microenvironment of RA. We found that the RA specimens contained higher levels of leukocyte subtypes, such as plasma cells and CD8 T cells, than normal tissue using the Wilcox test. The influx of immune cells into joint forms a complicated inflammatory network that activates fibroblast-like synoviocytes (FLS), inducing synovial vascular proliferation, and recruiting more immune cells to inflammatory sites(Chen et al. 2019).

We identified five clusters in the PPI network and the most significant one contained nine hub genes, five of which were confirmed by ROC curves analysis and RT-qPCR, including CCL25, CXCL9, CXCL10, CXCL11, and CXCL13. Chemokines can be divided into homeostatic subgroup (e.g., CXCL12) and inflammatory subgroup (such as CXCL9, CXCL10) depending on their function(Vergunst \& Tak 2005). However, this functional classification is not static, and chemokines act in a context-dependent manner based on different tissues and disease stages. A recent study found that plasma levels of chemokines, such as CXCL9, CXCL10, and CXCL13, 318 are much higher in patients with early untreated RA than in normal subjects. Among these 
319

320

321

322

323

324

325

326

327

328

329

330

331

332

333

334

335

336

337

338

339

340

341

342

343

344

345

discriminators, CXCL10 is associated with indicators of disease activity (e.g., DAS28), which may help determine whether RA patients are in an active phase of the disease(Pandya et al. 2017). CXCL10 is mainly produced by interferon- $\gamma$ stimulated $\mathrm{T}$ lymphocytes, neutrophils, and monocytes, and is therefore also known as $10 \mathrm{kDa}$ IP-10, which exerts its biological function by binding to the receptor CXCR3(Antonelli et al. 2014). High levels of CXCL10 in humoral components, such as peripheral blood and joint fluid, are typical of various autoimmune diseases, especially those with a predominance of Th1 cells(Lee et al. 2009). On the one hand, CXCL10 and CXCR3 play an important role in the homing of leukocytes to inflamed tissues(Romagnani et al. 2002); on the other hand, the massive accumulation of inflammatory cells, especially Th1 lymphocytes, enhances the secretion of IFN- $\gamma$ and TNF- $\alpha$, which in turn stimulates CXCL10 production, thus amplifying the feedback loop and leading to persistent inflammation and tissue damage(Antonelli et al. 2008). Chemokine receptors, which bind to chemokines, have also been shown to be involved in Th1-type and Th2-type inflammatory responses(Norii et al. 2006). Specific or non-specific targeting of chemokines and their receptors can be used to treat RA, and many targeted therapeutics have been tested in clinical trials with satisfactory results. For instance, patients taking both methotrexate and MDX1100, an antagonist of CXCL10, achieved reduced disease activity and improved symptom relief(Yellin et al. 2012). The application of drugs targeting chemokine receptors, such as $\mathrm{CP}-481,715$, showed promising results by reducing the number of macrophages in synovial tissue to ameliorate inflammatory response(Clucas et al. 2007).

We obtained 72 miRNA-gene regulatory relationships in our analysis of miRNA-gene interaction, of which seven miRNAs possessed two target genes, including hsa-miR-449a, hsamiR-297, hsa-miR-203a-3p, hsa-miR-449b-5p, hsa-miR-34c-5p, hsa-miR-573, and hsa-miR-34a5p. miRNAs belong to non-coding RNAs, which regulate cellular activity by degrading mRNA or inhibiting transcriptional processes(Esmailzadeh et al. 2017). It has been reported that about $60 \%$ of genes in the human body are regulated by miRNAs, and a single miRNA usually possesses thousands of target genes, which means that abnormal miRNA function may cause pathological 
346

347

348

349

350

351

352

353

354

355

356

357

358

359

360

361

362

363

364

365

366

367

368

369

370

371

372

consequences such as immune-related diseases and certain types of tumor(Baltimore et al. 2008;

O'Connell et al. 2010b). Widely expressed in a variety of immune cells (dendritic cells, monocytes, lymphocytes, etc.), miR-34a plays an important role in regulating cell development, function and survival, which is an important hub in the regulatory network of T cells(Taheri et al. 2020). In this study, we found that miR-34a-5p was significantly down-regulated in RA patients compared to health controls. Similarly, it has been shown that miR-34a was not well-expressed in RA synoviocytes, which contributed to their resistance to apoptosis and thus caused persistent inflammation of synovial tissue(Hou et al. 2019; Niederer et al. 2012). In addition, Wu et al. (2021) found that miR-34a in extracellular vesicles (Evs) was also involved in the development of RA(Wu et al. 2021). Elevated expression of miR-34a in BM-MSC-derived Evs activated the p53 signaling pathway by inhibiting cyclin I (CCNI) expression, which in turn inhibited FLS proliferation and promotes apoptosis, and thus had a positive effect on alleviating the inflammatory state of RA. However, some scholars hold the opposite view. It has been shown that miR-34a expression was up-regulated in PBMCs from RA patients(Ebrahimiyan et al. 2019; Xie et al. 2019), and silencing miR-34a markedly reduced release of inflammatory mediators(Kurowska-Stolarska et al. 2017). In other autoimmune diseases, such as Sjögren Syndrome, the level of miR-34a was also significantly elevated in patients(Kim et al. 2019). This controversy suggests that research on miR34a should continue. miR-449a has recently been identified as an important regulator of RA pathogenesis by suppressing HMGB1 and YY1 expression, thereby attenuating synovial hyperplasia, FLS migration, and the release of inflammatory mediators(Cai et al. 2019). Jin et al. (2018) found that miR-34c expression was significantly decreased in serum of Treg-depleted mice, an animal model commonly used in studies of autoimmune diseases(Jin et al. 2018).

Our study has limitations, including our focus on the most critical turquoise model in the process of co-expression network analysis, without adequate discussion of the black, blue, and purple modules, which were also strongly associated with RA. Our study size was small, and indepth clinical studies are needed to support our findings. 
In summary, we identified diagnostic genes and vital microRNAs associated with RA, which can help us to better understand the pathogenesis behind this disorder and provide theoretical support for exploring more effective therapies.

\section{References}

Antonelli A, Ferrari SM, Giuggioli D, Ferrannini E, Ferri C, and Fallahi P. 2014. Chemokine (C-X-C motif) ligand (CXCL)10 in autoimmune diseases. Autoimmun Rev 13:272-280. 10.1016/j.autrev.2013.10.010

Antonelli A, Ferri C, Ferrari SM, Colaci M, and Fallahi P. 2008. Immunopathogenesis of HCV-related endocrine manifestations in chronic hepatitis and mixed cryoglobulinemia. Autoimmun Rev 8:18-23. 10.1016/j.autrev.2008.07.017

Bader GD, and Hogue CW. 2003. An automated method for finding molecular complexes in large protein interaction networks. BMC Bioinformatics 4:2. 10.1186/1471-2105-4-2

Baltimore D, Boldin MP, O'Connell RM, Rao DS, and Taganov KD. 2008. MicroRNAs: new regulators of immune cell development and function. Nat Immunol 9:839-845. 10.1038/ni.f.209

Bertolini DR, Nedwin GE, Bringman TS, Smith DD, and Mundy GR. 1986. Stimulation of bone resorption and inhibition of bone formation in vitro by human tumour necrosis factors. Nature 319:516-518.

Boyle DL, Soma K, Hodge J, Kavanaugh A, Mandel D, Mease P, Shurmur R, Singhal AK, Wei N, Rosengren S, Kaplan I, Krishnaswami S, Luo Z, Bradley J, and Firestein GS. 2015. The JAK inhibitor tofacitinib suppresses synovial JAK1-STAT signalling in rheumatoid arthritis. Ann Rheum Dis 74:1311-1316. 10.1136/annrheumdis-2014-206028

Cai Y, Jiang C, Zhu J, Xu K, Ren X, Xu L, Hu P, Wang B, Yuan Q, Guo Y, Sun J, Xu P, and Qiu Y. 2019. miR-449a inhibits cell proliferation, migration, and inflammation by regulating high-mobility group box protein 1 and forms a mutual inhibition loop with Yin Yang 1 in rheumatoid arthritis fibroblast-like synoviocytes. Arthritis Res Ther 21:134. 10.1186/s13075-019-1920-0

Chen J, Wright K, Davis JM, Jeraldo P, Marietta EV, Murray J, Nelson H, Matteson EL, and Taneja V. 2016. An expansion of rare lineage intestinal microbes characterizes rheumatoid arthritis. Genome Med 8:43. 10.1186/s13073-016-0299-7

Chen Y, and Wang X. 2020. miRDB: an online database for prediction of functional microRNA targets. Nucleic Acids Res 48:D127-d131. 10.1093/nar/gkz757

Chen Z, Bozec A, Ramming A, and Schett G. 2019. Anti-inflammatory and immune-regulatory cytokines in rheumatoid arthritis. Nat Rev Rheumatol 15:9-17. 10.1038/s41584-018-0109-2

Cline MS, Smoot M, Cerami E, Kuchinsky A, Landys N, Workman C, Christmas R, Avila-Campilo I, Creech M, Gross B, Hanspers K, Isserlin R, Kelley R, Killcoyne S, Lotia S, Maere S, Morris J, Ono K, Pavlovic V, Pico AR, Vailaya A, Wang P-L, Adler A, Conklin BR, Hood L, Kuiper M, Sander C, Schmulevich I, Schwikowski B, Warner GJ, Ideker T, and Bader GD. 2007. Integration of biological networks and gene expression data using Cytoscape. Nature Protocols 2:2366-2382. 10.1038/nprot.2007.324

Clucas AT, Shah A, Zhang YD, Chow VF, and Gladue RP. 2007. Phase I evaluation of the safety, pharmacokinetics and pharmacodynamics of CP-481,715. Clinical pharmacokinetics 46:757-766.

Ebrahimiyan H, Rezaei N, Vojdanian M, Aslani S, Jamshidi A, and Mahmoudi M. 2019. microRNA involvement in

Peer) reviewing PDF | (2020:11:55737:2:0:NEW 29 Mar 2021) 
412

413

414

415

416

417

418

419

420

421

422

423

424

425

426

427

428

429

430

431

432

433

434

435

436

437

438

439

440

441

442

443

444

445

446

447

448

449

450

451

452

the regulation of survivin in peripheral blood mononuclear cells from rheumatoid arthritis patients. Int $J$ Rheum Dis 22:1107-1114. 10.1111/1756-185x.13520

Esmailzadeh S, Mansoori B, Mohammadi A, and Baradaran B. 2017. Regulatory roles of micro-RNAs in T cell autoimmunity. Immunol Invest 46:864-879. 10.1080/08820139.2017.1373901

Hajishengallis G. 2015. Periodontitis: from microbial immune subversion to systemic inflammation. Nat Rev Immunol 15:30-44. 10.1038/nri3785

Hirayama T, Dai S, Abbas S, Yamanaka Y, and Abu-Amer Y. 2005. Inhibition of inflammatory bone erosion by constitutively active STAT-6 through blockade of JNK and NF- $\mathrm{kB}$ activation. Arthritis \& Rheumatism 52:2719-2729.

Hou C, Wang D, and Zhang L. 2019. MicroRNA-34a-3p inhibits proliferation of rheumatoid arthritis fibroblast-like synoviocytes. Mol Med Rep 20:2563-2570. 10.3892/mmr.2019.10516

Jin F, Hu H, Xu M, Zhan S, Wang Y, Zhang H, and Chen X. 2018. Serum microRNA Profiles Serve as Novel Biomarkers for Autoimmune Diseases. Front Immunol 9:2381. 10.3389/fimmu.2018.02381

Kawatkar A, Portugal C, Chu L-H, and Iyer R. 2012. Racial/Ethnic Trends in Incidence and Prevalence of Rheumatoid Arthritis in a Large Multi-Ethnic Managed Care Population.: 2514. Arthritis \& Rheumatism 64.

Kharlamova N, Jiang X, Sherina N, Potempa B, Israelsson L, Quirke AM, Eriksson K, Yucel-Lindberg T, Venables PJ, Potempa J, Alfredsson L, and Lundberg K. 2016. Antibodies to Porphyromonas gingivalis Indicate Interaction Between Oral Infection, Smoking, and Risk Genes in Rheumatoid Arthritis Etiology. Arthritis Rheumatol 68:604-613. 10.1002/art.39491

Kim YJ, Yeon Y, Lee WJ, Shin YU, Cho H, Sung YK, Kim DR, Lim HW, and Kang MH. 2019. Comparison of MicroRNA Expression in Tears of Normal Subjects and Sjögren Syndrome Patients. Invest Ophthalmol Vis Sci 60:4889-4895. 10.1167/iovs.19-27062

Kurowska-Stolarska M, Alivernini S, Melchor EG, Elmesmari A, Tolusso B, Tange C, Petricca L, Gilchrist DS, Di Sante G, Keijzer C, Stewart L, Di Mario C, Morrison V, Brewer JM, Porter D, Milling S, Baxter RD, McCarey D, Gremese E, Lemke G, Ferraccioli G, McSharry C, and McInnes IB. 2017. MicroRNA-34a dependent regulation of AXL controls the activation of dendritic cells in inflammatory arthritis. Nat Commun 8:15877. 10.1038/ncomms 15877

Lackie J. 2010. A dictionary of biomedicine: Oxford University Press.

Langfelder P, and Horvath S. 2008. WGCNA: an R package for weighted correlation network analysis. $B M C$ Bioinformatics 9:559. 10.1186/1471-2105-9-559

Langfelder P, and Horvath S. 2012. Fast R Functions for Robust Correlations and Hierarchical Clustering. J Stat Softw 46.

Lee EY, Lee ZH, and Song YW. 2009. CXCL10 and autoimmune diseases. Autoimmun Rev 8:379-383. 10.1016/j.autrev.2008.12.002

Leng RX, Pan HF, Qin WZ, Chen GM, and Ye DQ. 2011. Role of microRNA-155 in autoimmunity. Cytokine Growth Factor Rev 22:141-147. 10.1016/j.cytogfr.2011.05.002

Liberzon A, Birger C, Thorvaldsdottir H, Ghandi M, Mesirov JP, and Tamayo P. 2015. The Molecular Signatures Database (MSigDB) hallmark gene set collection. Cell Syst 1:417-425. 10.1016/j.cels.2015.12.004

Liberzon A, Subramanian A, Pinchback R, Thorvaldsdottir H, Tamayo P, and Mesirov JP. 2011. Molecular signatures database (MSigDB) 3.0. Bioinformatics 27:1739-1740. 10.1093/bioinformatics/btr260

Liu W, and Wang X. 2019. Prediction of functional microRNA targets by integrative modeling of microRNA binding 
and target expression data. Genome Biol 20:18. 10.1186/s13059-019-1629-z

MacGregor AJ, Snieder H, Rigby AS, Koskenvuo M, Kaprio J, Aho K, and Silman AJ. 2000. Characterizing the quantitative genetic contribution to rheumatoid arthritis using data from twins. Arthritis Rheum 43:30-37. 10.1002/1529-0131(200001)43:1<30::Aid-anr5>3.0.Co;2-b

Mootha VK, Lindgren CM, Eriksson KF, Subramanian A, Sihag S, Lehar J, Puigserver P, Carlsson E, Ridderstrale M, Laurila E, Houstis N, Daly MJ, Patterson N, Mesirov JP, Golub TR, Tamayo P, Spiegelman B, Lander ES, Hirschhorn JN, Altshuler D, and Groop LC. 2003. PGC-1alpha-responsive genes involved in oxidative phosphorylation are coordinately downregulated in human diabetes. Nat Genet 34:267-273. 10.1038/ng1180

Newman AM, Liu CL, Green MR, Gentles AJ, Feng W, Xu Y, Hoang CD, Diehn M, and Alizadeh AA. 2015. Robust enumeration of cell subsets from tissue expression profiles. Nat Methods 12:453-457. 10.1038/nmeth.3337

Niederer F, Trenkmann M, Ospelt C, Karouzakis E, Neidhart M, Stanczyk J, Kolling C, Gay RE, Detmar M, Gay S, Jüngel A, and Kyburz D. 2012. Down-regulation of microRNA-34a* in rheumatoid arthritis synovial fibroblasts promotes apoptosis resistance. Arthritis Rheum 64:1771-1779. 10.1002/art.34334

Norii M, Yamamura M, Iwahashi M, Ueno A, Yamana J, and Makino H. 2006. Selective recruitment of CXCR3+ and CCR5+ CCR4+ T cells into synovial tissue in patients with rheumatoid arthritis. Acta Medica Okayama 60:149-157.

O'connell RM, Rao DS, Chaudhuri AA, and Baltimore D. 2010a. Physiological and pathological roles for microRNAs in the immune system. Nature Reviews Immunology 10:111-122.

O'Connell RM, Rao DS, Chaudhuri AA, and Baltimore D. 2010b. Physiological and pathological roles for microRNAs in the immune system. Nat Rev Immunol 10:111-122. 10.1038/nri2708

Ohshima S, Saeki Y, Mima T, Sasai M, Nishioka K, Nomura S, Kopf M, Katada Y, Tanaka T, and Suemura M. 1998. Interleukin 6 plays a key role in the development of antigen-induced arthritis. Proceedings of the National Academy of Sciences 95:8222-8226.

Otasek D, Morris JH, Boucas J, Pico AR, and Demchak B. 2019. Cytoscape Automation: empowering workflowbased network analysis. Genome Biol 20:185. 10.1186/s13059-019-1758-4

Pan W, Zhu S, Yuan M, Cui H, Wang L, Luo X, Li J, Zhou H, Tang Y, and Shen N. 2010. MicroRNA-21 and microRNA-148a contribute to DNA hypomethylation in lupus CD4+ T cells by directly and indirectly targeting DNA methyltransferase 1. J Immunol 184:6773-6781. 10.4049/jimmunol.0904060

Pandya JM, Lundell AC, Andersson K, Nordstrom I, Theander E, and Rudin A. 2017. Blood chemokine profile in untreated early rheumatoid arthritis: CXCL10 as a disease activity marker. Arthritis Res Ther 19:20. 10.1186/s13075-017-1224-1

Ritchie ME, Phipson B, Wu D, Hu Y, Law CW, Shi W, and Smyth GK. 2015. limma powers differential expression analyses for RNA-sequencing and microarray studies. Nucleic Acids Res 43:e47. 10.1093/nar/gkv007

Robin X, Turck N, Hainard A, Tiberti N, Lisacek F, Sanchez JC, and Muller M. 2011. pROC: an open-source package for R and $\mathrm{S}+$ to analyze and compare ROC curves. BMC Bioinformatics 12:77. 10.1186/1471-2105-12-77

Romagnani P, Rotondi M, Lazzeri E, Lasagni L, Francalanci M, Buonamano A, Milani S, Vitti P, Chiovato L, Tonacchera M, Bellastella A, and Serio M. 2002. Expression of IP-10/CXCL10 and MIG/CXCL9 in the thyroid and increased levels of IP-10/CXCL10 in the serum of patients with recent-onset Graves' disease. $\mathrm{Am}$ J Pathol 161:195-206. 10.1016/s0002-9440(10)64171-5

Schett G, Elewaut D, McInnes IB, Dayer J-M, and Neurath MF. 2013. How cytokine networks fuel inflammation: toward a cytokine-based disease taxonomy. Nature medicine 19:822-824. 
Shannon P, Markiel A, Ozier O, Baliga NS, Wang JT, Ramage D, Amin N, Schwikowski B, and Ideker T. 2003. Cytoscape: a software environment for integrated models of biomolecular interaction networks. Genome Res 13:2498-2504. 10.1101/gr.1239303

Simpson LJ, and Ansel KM. 2015. MicroRNA regulation of lymphocyte tolerance and autoimmunity. The Journal of clinical investigation 125:2242-2249.

Smolen JS, Aletaha D, and McInnes IB. 2016. Rheumatoid arthritis. Lancet 388:2023-2038. 10.1016/s01406736(16)30173-8

Subramanian A, Tamayo P, Mootha VK, Mukherjee S, Ebert BL, Gillette MA, Paulovich A, Pomeroy SL, Golub TR, Lander ES, and Mesirov JP. 2005. Gene set enrichment analysis: a knowledge-based approach for interpreting genome-wide expression profiles. Proc Natl Acad Sci U S A 102:15545-15550. 10.1073/pnas.0506580102

Szklarczyk D, Franceschini A, Wyder S, Forslund K, Heller D, Huerta-Cepas J, Simonovic M, Roth A, Santos A, Tsafou KP, Kuhn M, Bork P, Jensen LJ, and von Mering C. 2015. STRING v10: protein-protein interaction networks, integrated over the tree of life. Nucleic Acids Res 43:D447-452. 10.1093/nar/gku1003

Szklarczyk D, Gable AL, Lyon D, Junge A, Wyder S, Huerta-Cepas J, Simonovic M, Doncheva NT, Morris JH, Bork P, Jensen LJ, and Mering CV. 2019. STRING v11: protein-protein association networks with increased coverage, supporting functional discovery in genome-wide experimental datasets. Nucleic Acids Res 47:D607-d613. 10.1093/nar/gky1131

Szklarczyk D, Morris JH, Cook H, Kuhn M, Wyder S, Simonovic M, Santos A, Doncheva NT, Roth A, Bork P, Jensen LJ, and von Mering C. 2017. The STRING database in 2017: quality-controlled protein-protein association networks, made broadly accessible. Nucleic Acids Res 45:D362-d368. 10.1093/nar/gkw937

Taheri F, Ebrahimi SO, Shareef S, and Reiisi S. 2020. Regulatory and immunomodulatory role of miR-34a in T cell immunity. Life Sci 262:118209. 10.1016/j.lfs.2020.118209

Vergunst CE, and Tak PP. 2005. Chemokines: their role in rheumatoid arthritis. Current rheumatology reports 7:382388.

Wu H, Zhou X, Wang X, Cheng W, Hu X, Wang Y, Luo B, Huang W, and Gu J. 2021. miR-34a in extracellular vesicles from bone marrow mesenchymal stem cells reduces rheumatoid arthritis inflammation via the cyclin I/ATM/ATR/p53 axis. J Cell Mol Med 25:1896-1910. 10.1111/jcmm.15857

Xie M, Wang J, Gong W, Xu H, Pan X, Chen Y, Ru S, Wang H, Chen X, Zhao Y, Li J, Yin Q, Xia S, Zhou X, Liu X, and Shao Q. 2019. NF-kappaB-driven miR-34a impairs Treg/Th17 balance via targeting Foxp3. J Autoimmun 102:96-113. 10.1016/j.jaut.2019.04.018

Yang H, and Li H. 2019. CD36 identified by weighted gene co-expression network analysis as a hub candidate gene in lupus nephritis. PeerJ 7:e7722. 10.7717/peerj.7722

Yao Q, Song Z, Wang B, Qin Q, and Zhang JA. 2019. Identifying Key Genes and Functionally Enriched Pathways in Sjogren's Syndrome by Weighted Gene Co-Expression Network Analysis. Front Genet 10:1142. 10.3389/fgene.2019.01142

Yellin M, Paliienko I, Balanescu A, Ter-Vartanian S, Tseluyko V, Xu LA, Tao X, Cardarelli PM, LeBlanc H, and Nichol G. 2012. A phase II, randomized, double-blind, placebo-controlled study evaluating the efficacy and safety of MDX-1100, a fully human anti-CXCL10 monoclonal antibody, in combination with methotrexate in patients with rheumatoid arthritis. Arthritis \& Rheumatism 64:1730-1739.

Yu G, Wang LG, Han Y, and He QY. 2012. clusterProfiler: an R package for comparing biological themes among 
535 gene clusters. Omics 16:284-287. 10.1089/omi.2011.0118

536 Zhang J, Wang X, Xu L, Zhang Z, Wang F, and Tang X. 2020. Investigation of Potential Genetic Biomarkers and Molecular Mechanism of Ulcerative Colitis Utilizing Bioinformatics Analysis. Biomed Res Int 2020:4921387. 10.1155/2020/4921387

Zhong D, Wu C, Bai J, Xu D, Zeng X, and Wang Q. 2020. Co-expression network analysis reveals the pivotal role of 541 mitochondrial dysfunction and interferon signature in juvenile dermatomyositis. PeerJ 8. 10.7717/peerj.8611 
Figure 1

Flow chart of the study design.

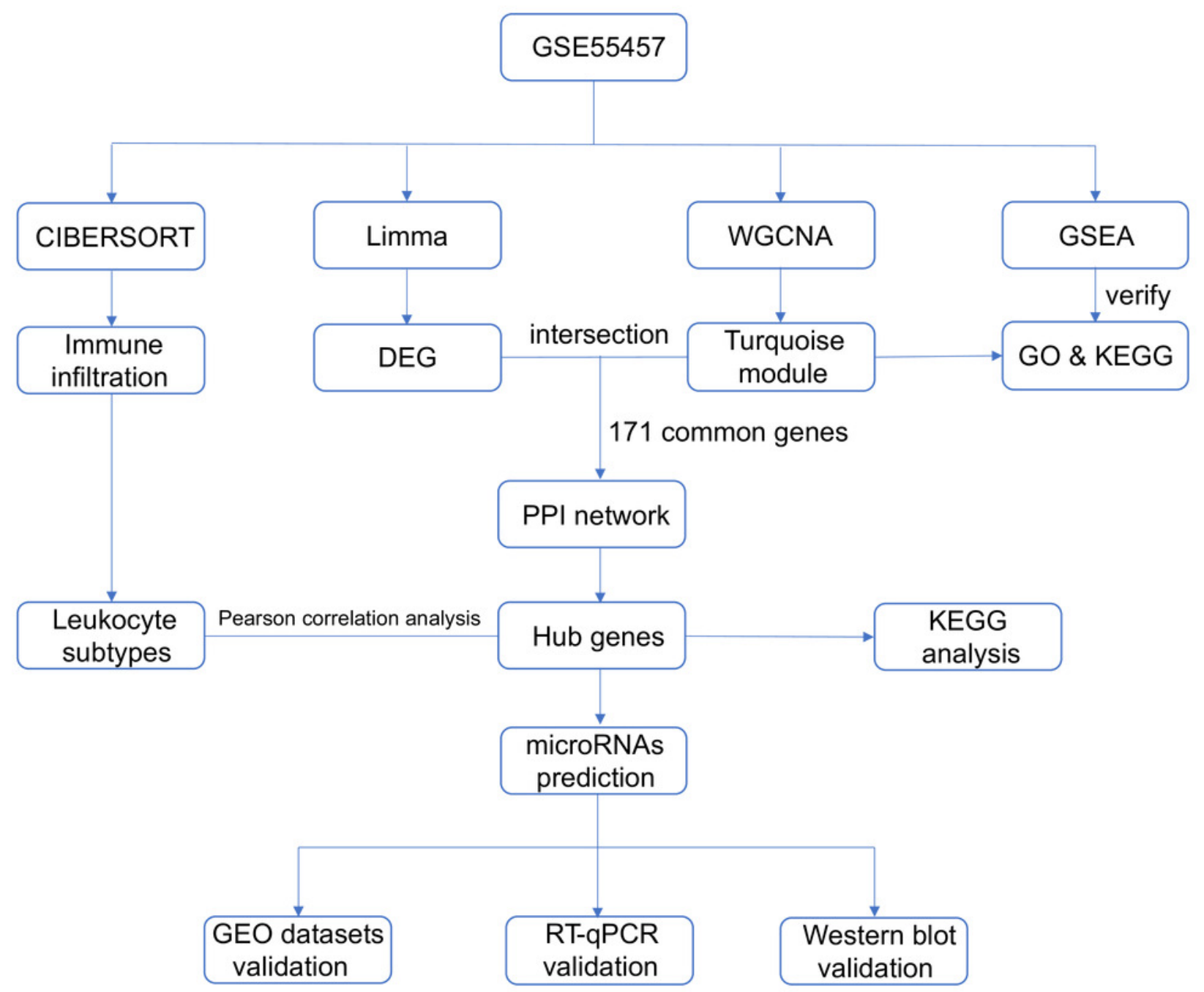


Figure 2

Visualization of differentially expressed genes (DEGs).

(A) DEGs filtered by thresholds were presented in volcano map. Red dots represent upregulated genes and blue dots represent down-regulated genes. (B) Heatmap showed the expression of DEGs in each sample.

A

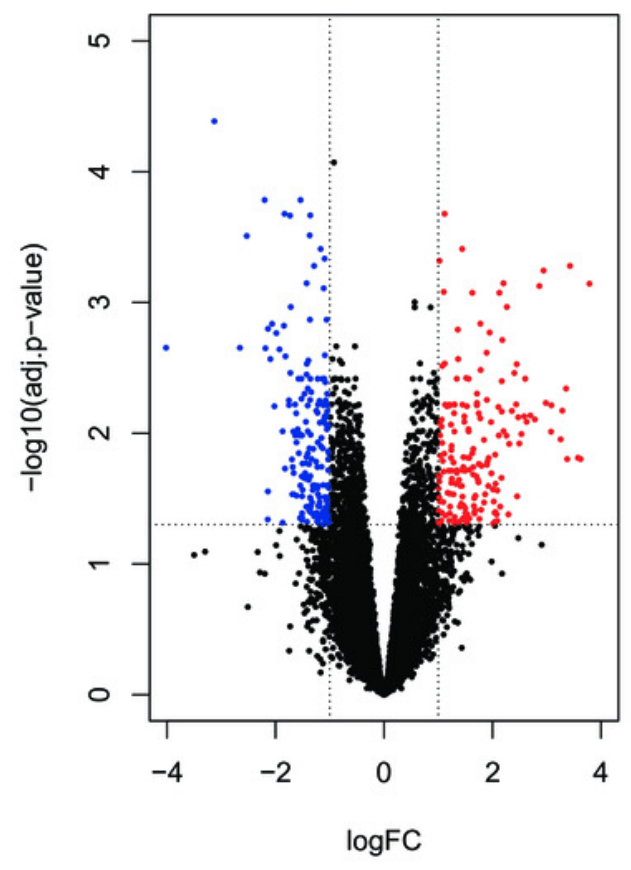

B

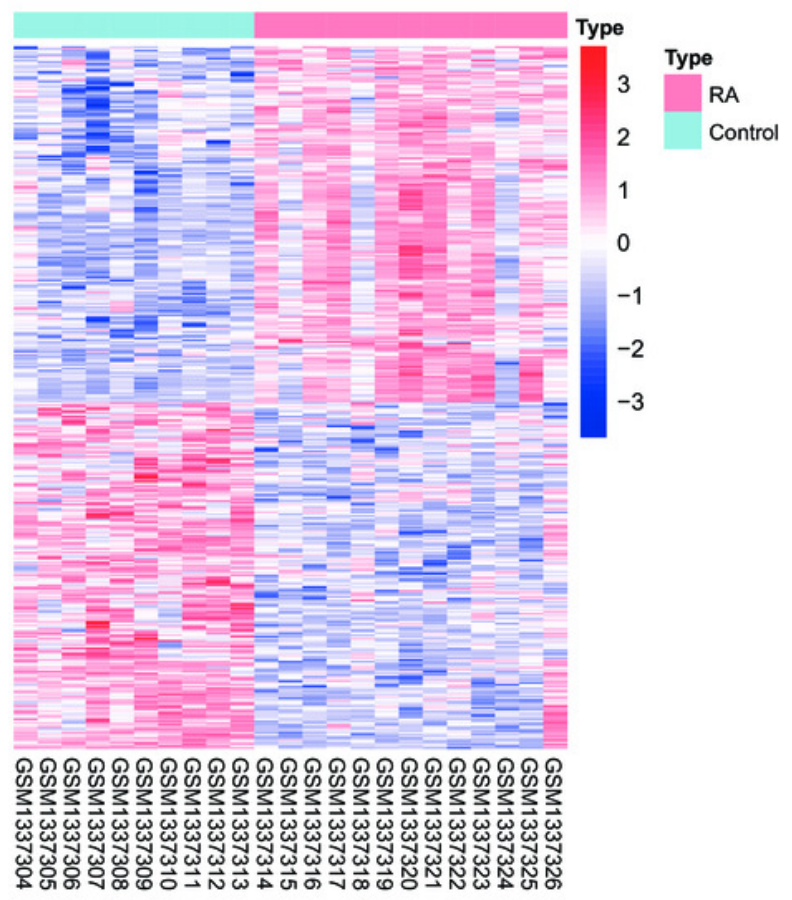




\section{Figure 3}

Identification of key module closely related to RA.

(A) Clustering of samples and removal of outliers. (B) $\beta=5$ was chosen as appropriate soft-

thresholding power, for which scale-free topology fit index $\left(R^{2}\right)$ reached 0.90. (C) Coexpression analysis resulted in 14 modules. Each branch represents one gene, and every color represents a co-expression module. (D) Number of genes in each co-expression module. (E) Heatmap showed the correlation between module eigengenes and clinical traits. (F) Gene significance (GS) and module membership (MM) of each gene in turquoise module. 
A

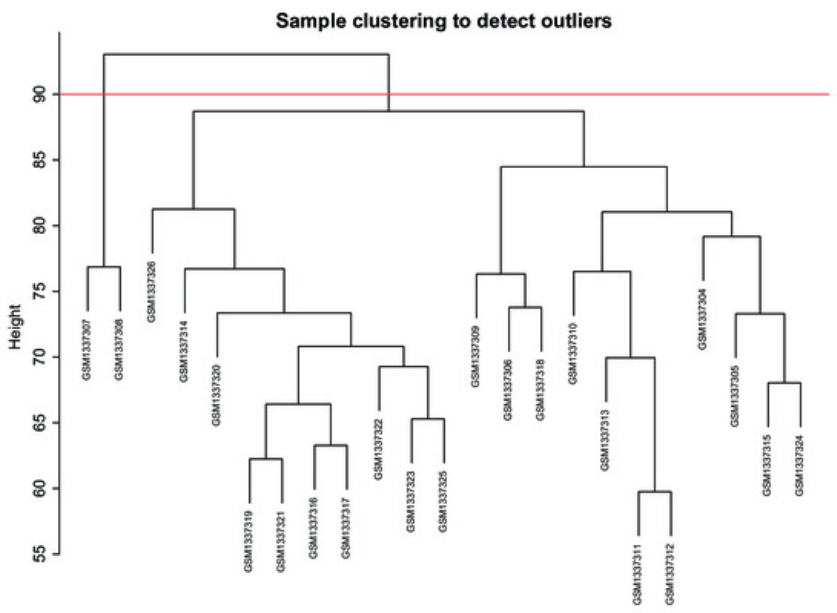

C

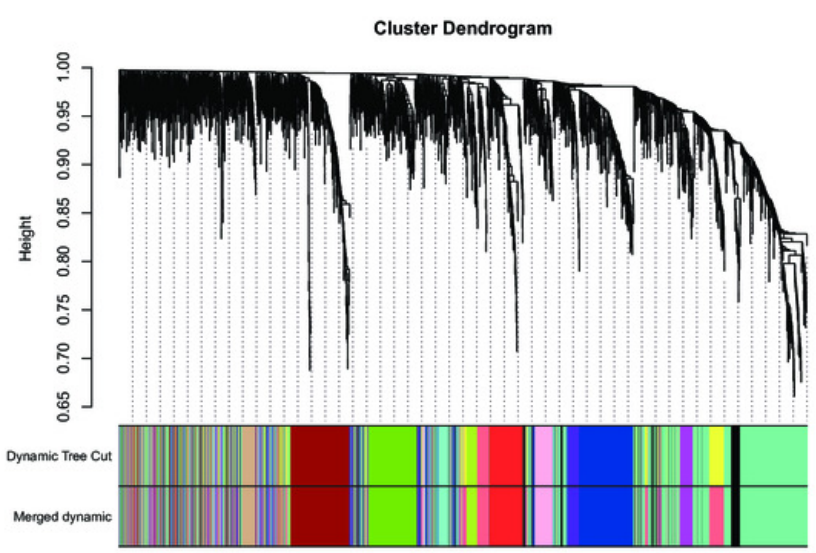

E

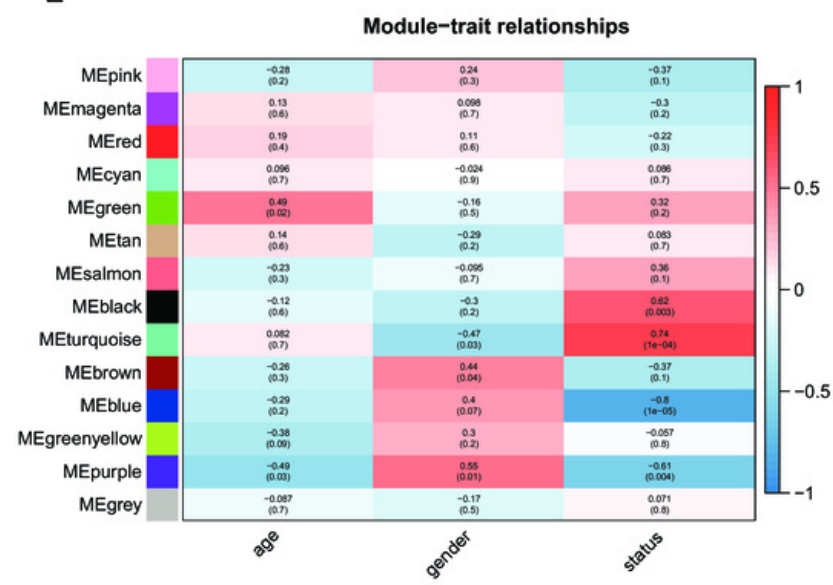

B
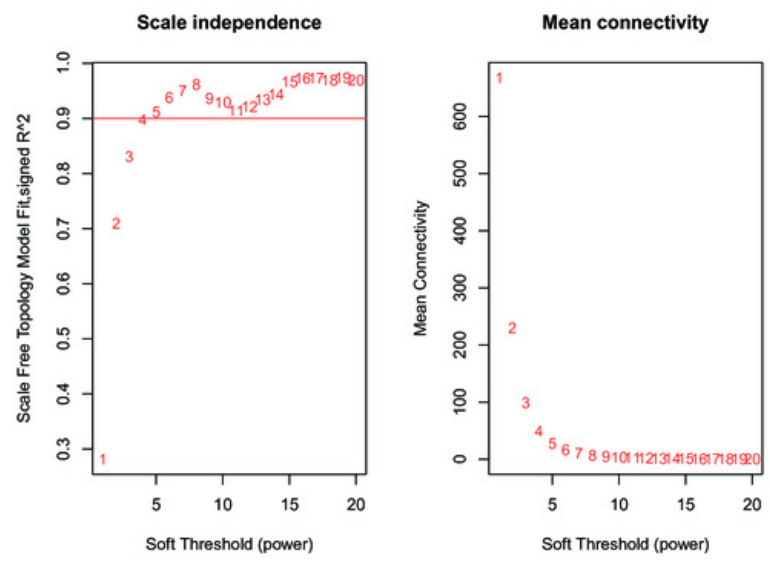

D

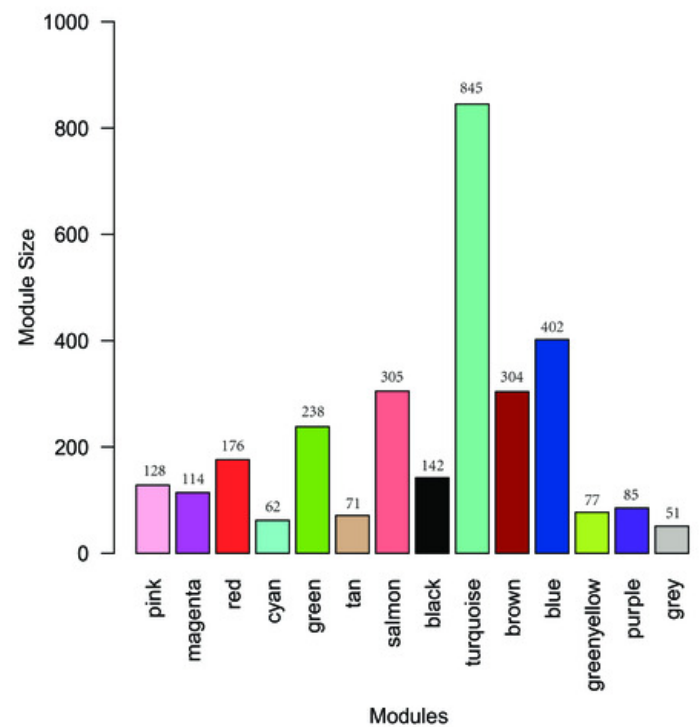

F

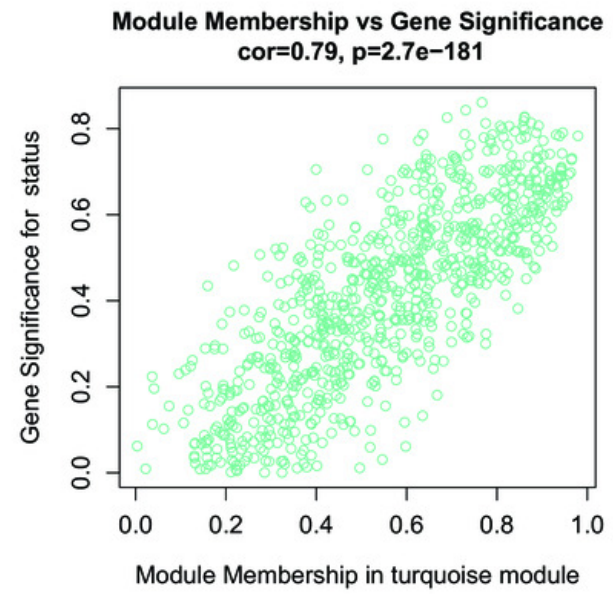


Figure 4

Results of functional enrichment analysis.

(A) GO analysis of turquoise module genes. (B) Circle plot was displayed to visualize correspondence between genes and top $3 \mathrm{GO}$ terms. (C) KEGG analysis of turquoise module genes. (D) Circle plot was displayed to visualize correspondence between genes and top 10 KEGG pathways. 
A

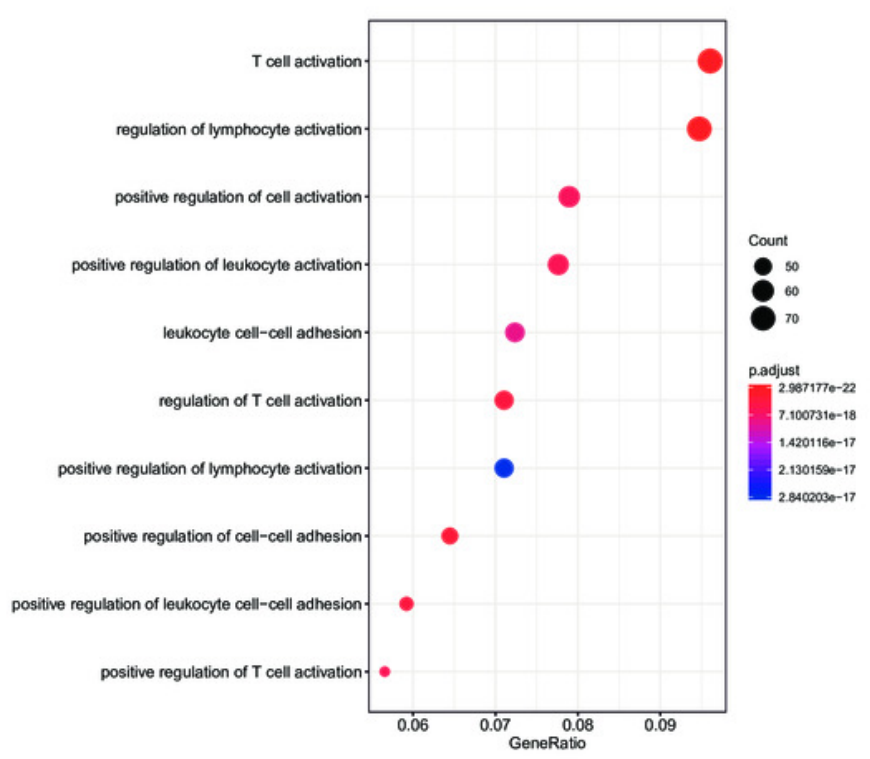

C

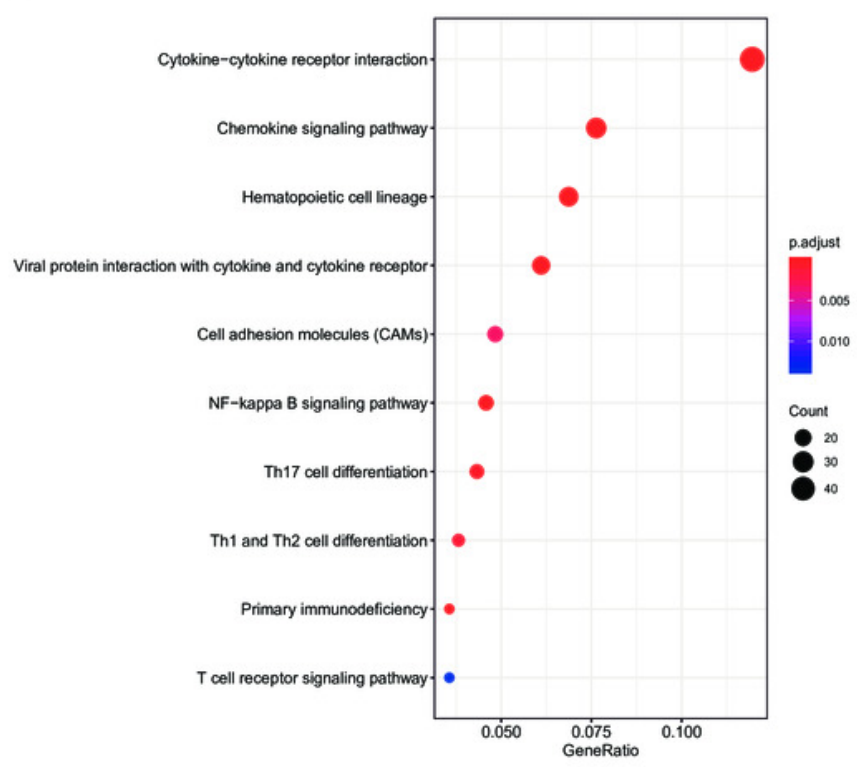

B

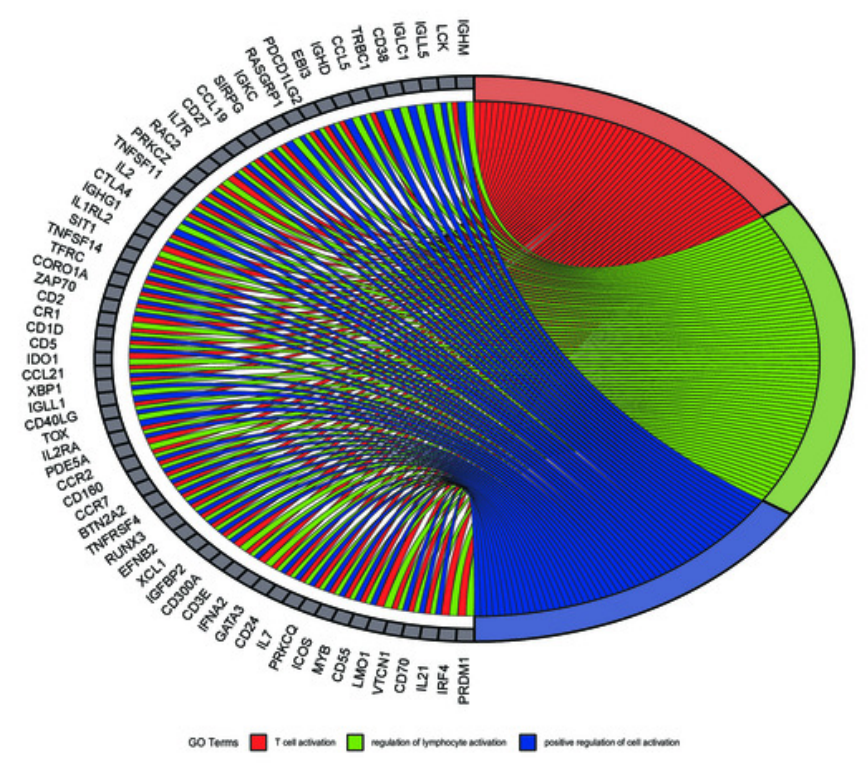

D

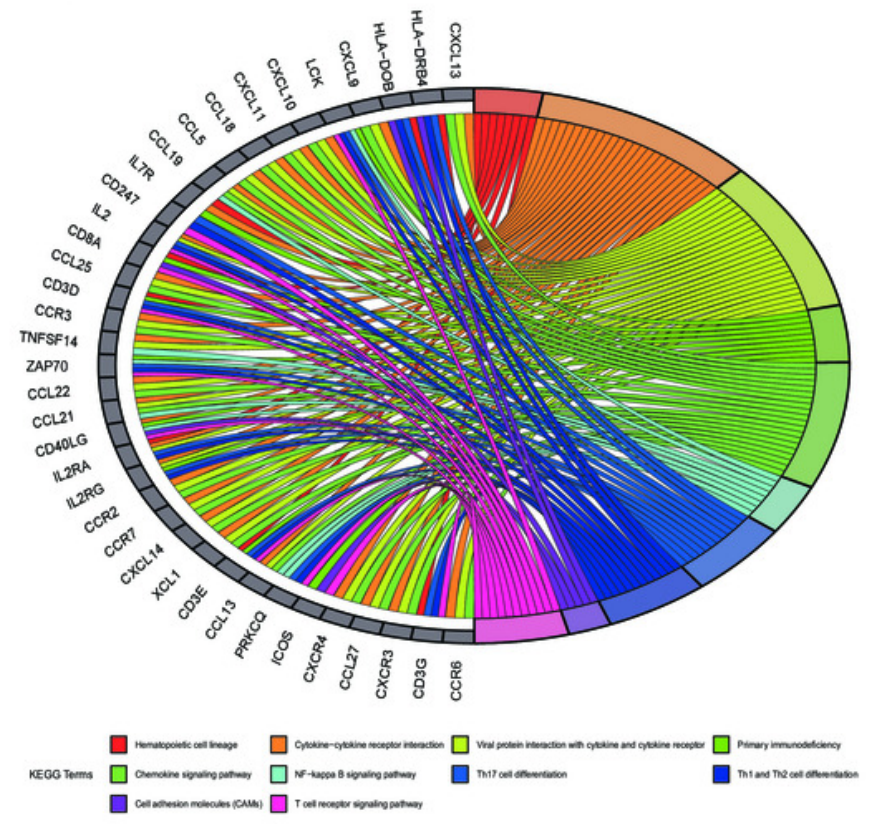


Figure 5

Results of GSEA analysis.

Enrichment plots for inflammatory response (A), IL6-JAK-STAT3 signaling (B), complement (C), interferon alpha response (D), interferon gamma response (E). 
A

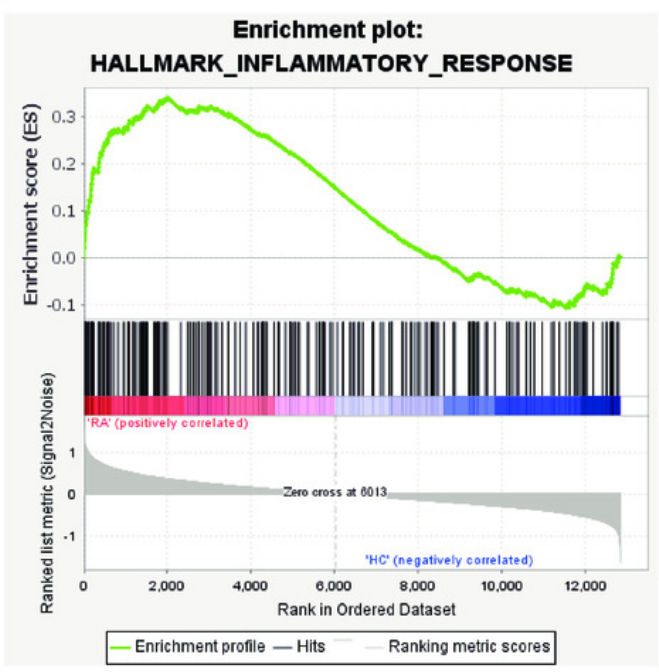

C

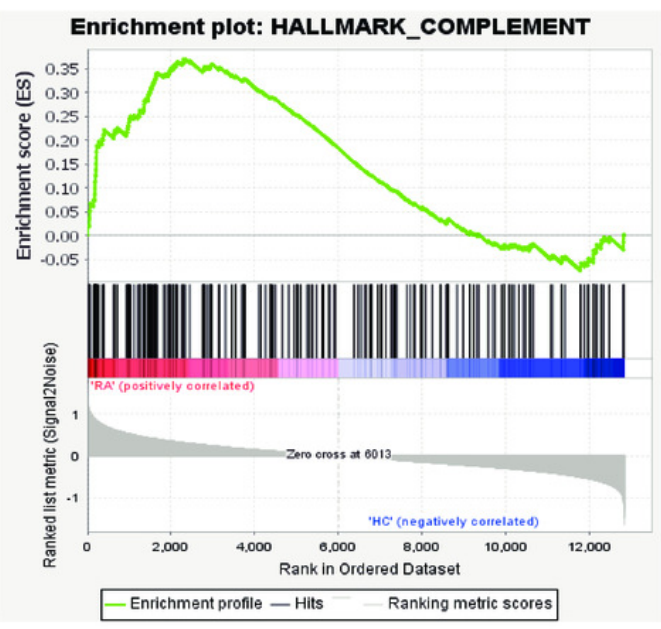

B

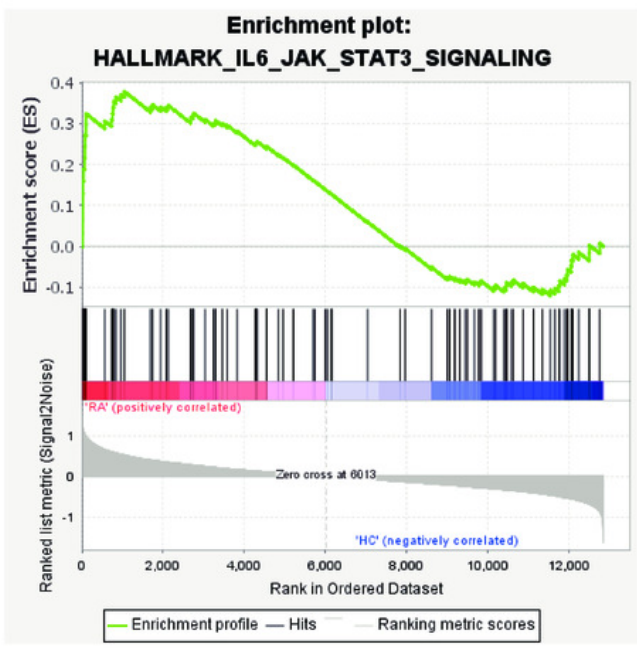

D

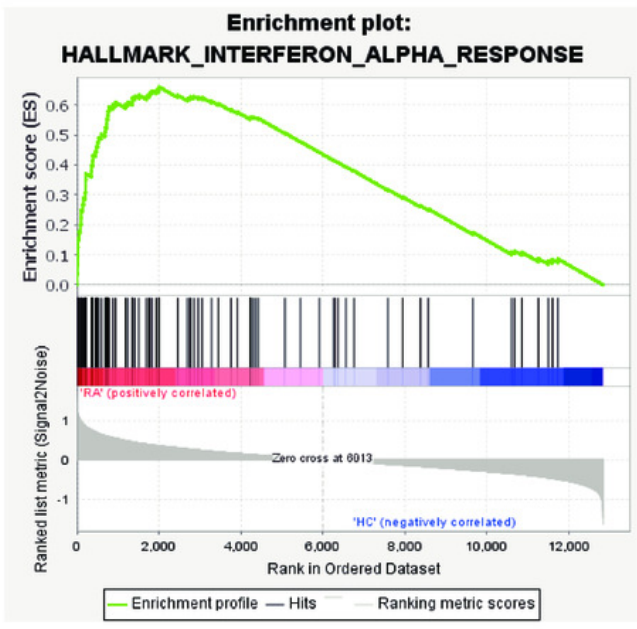

E Enrichment plot: HALLMARK_INTERFERON_GAMMA_RESPONSE

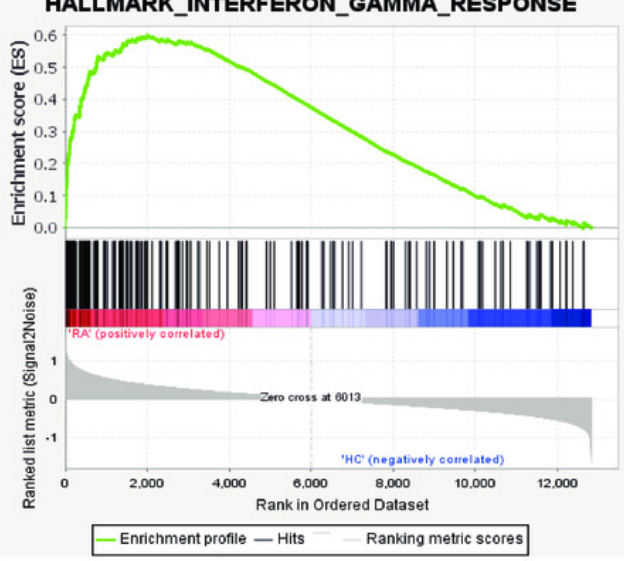


Figure 6

PPI network construction and mining hub genes.

(A) The intersection of DEGs and turquoise module genes was presented in Venn diagram.

(B) The PPI network of intersection genes was constructed in Cytoscape. Genes in the highest scoring cluster were marked in red. (C) KEGG analysis of nine hub genes. 
A

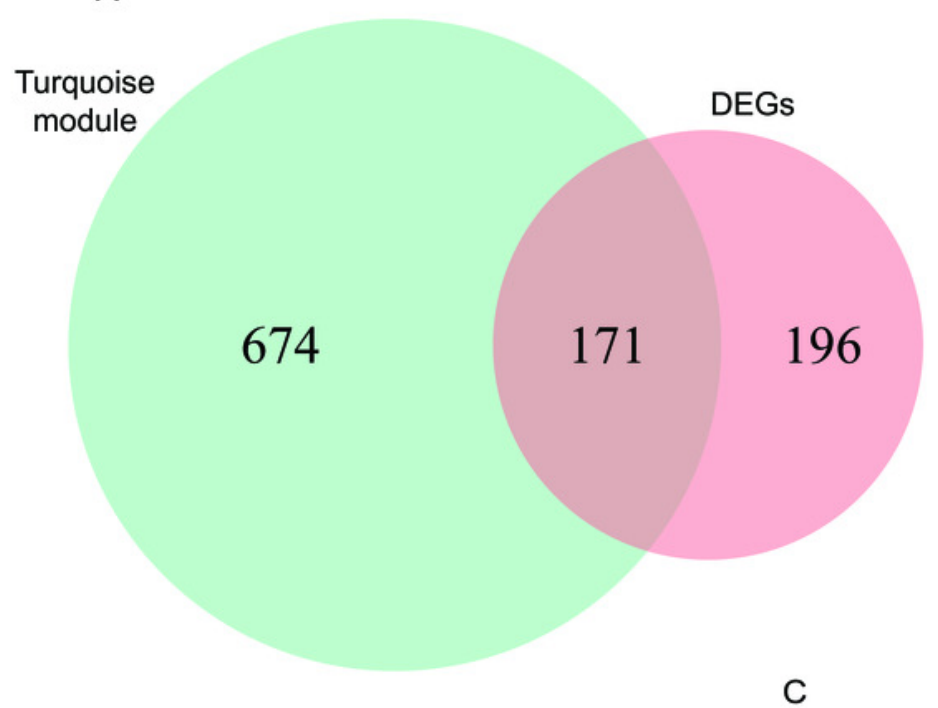

B

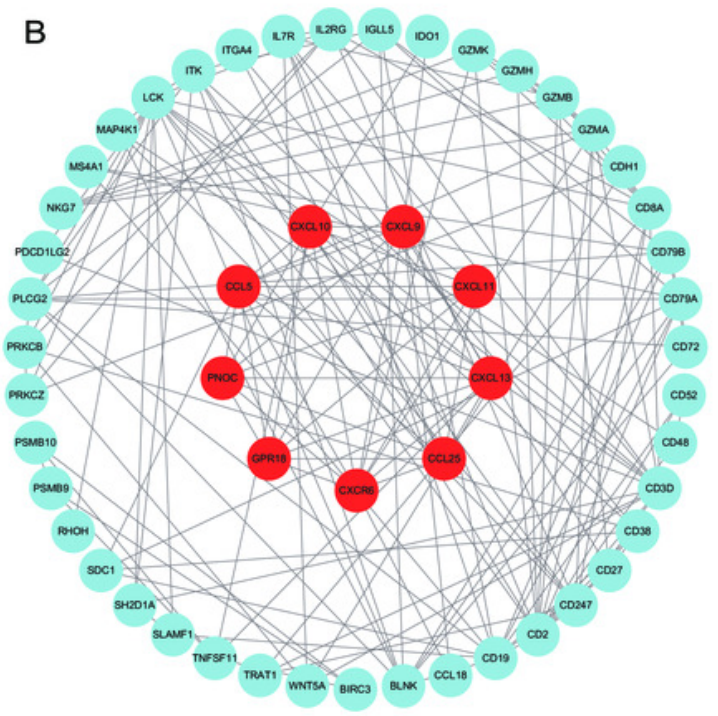

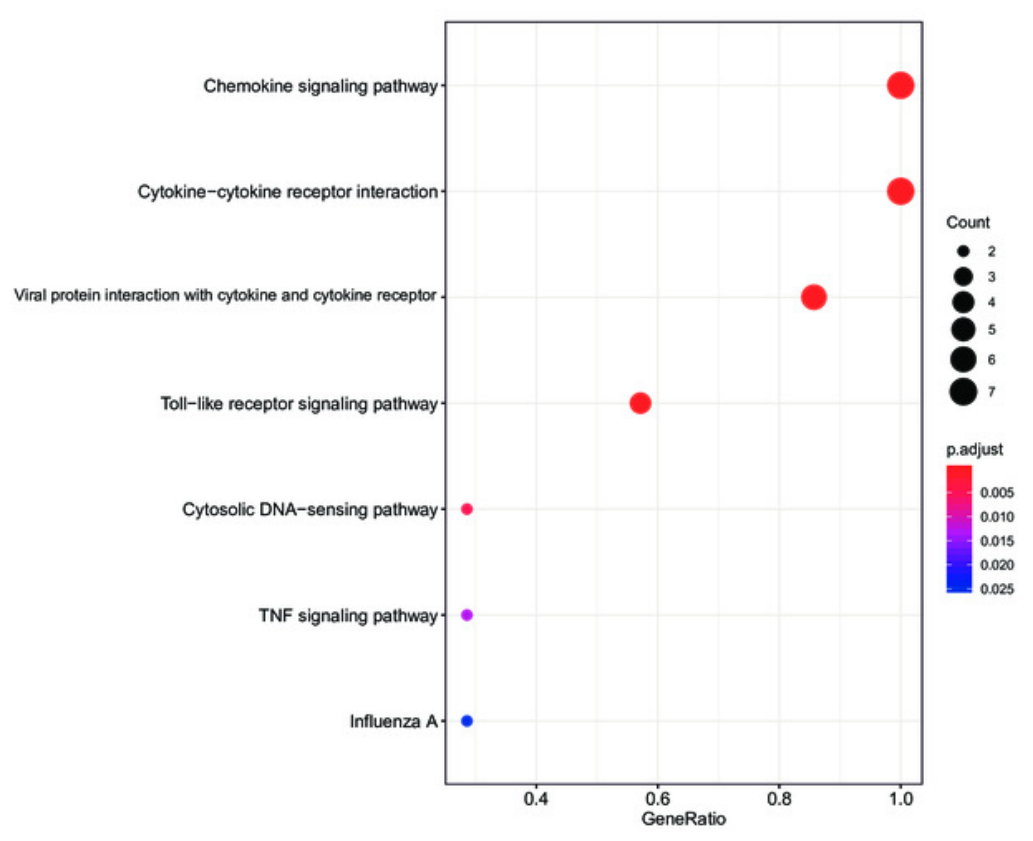




\section{Figure 7}

Immune infiltration analysis performed by CIBERSORT.

(A) Stacked bar chart showed contents of various immune cells in each sample. (B) The contents of 22 types of immune cells in normal (blue color) and RA (red color) groups were compared. P-value $<0.05$ was considered statistically significant. (C) Heat map of correlation between hub genes and leukocyte subtypes. The shades of color represent the magnitude of correlation coefficient. * p-value $<0.05 ; * *$-value $<0.01$.

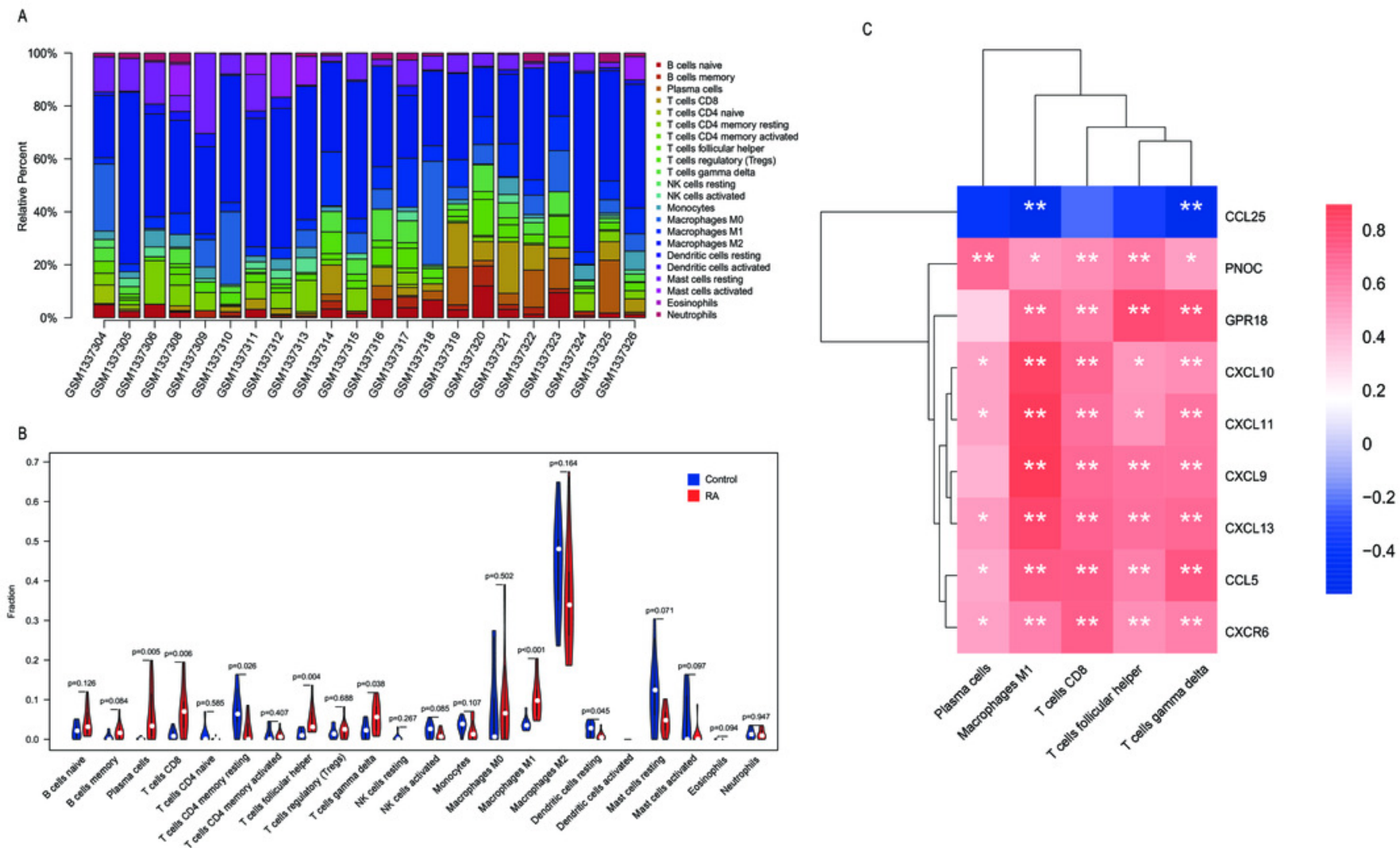




\section{Figure 8}

Interaction network between hub genes and miRNAs.

Hub genes were colored in red; miRNAs with one target gene were indicated by blue nodes; core miRNAs targeting two genes were indicated by green arrows.

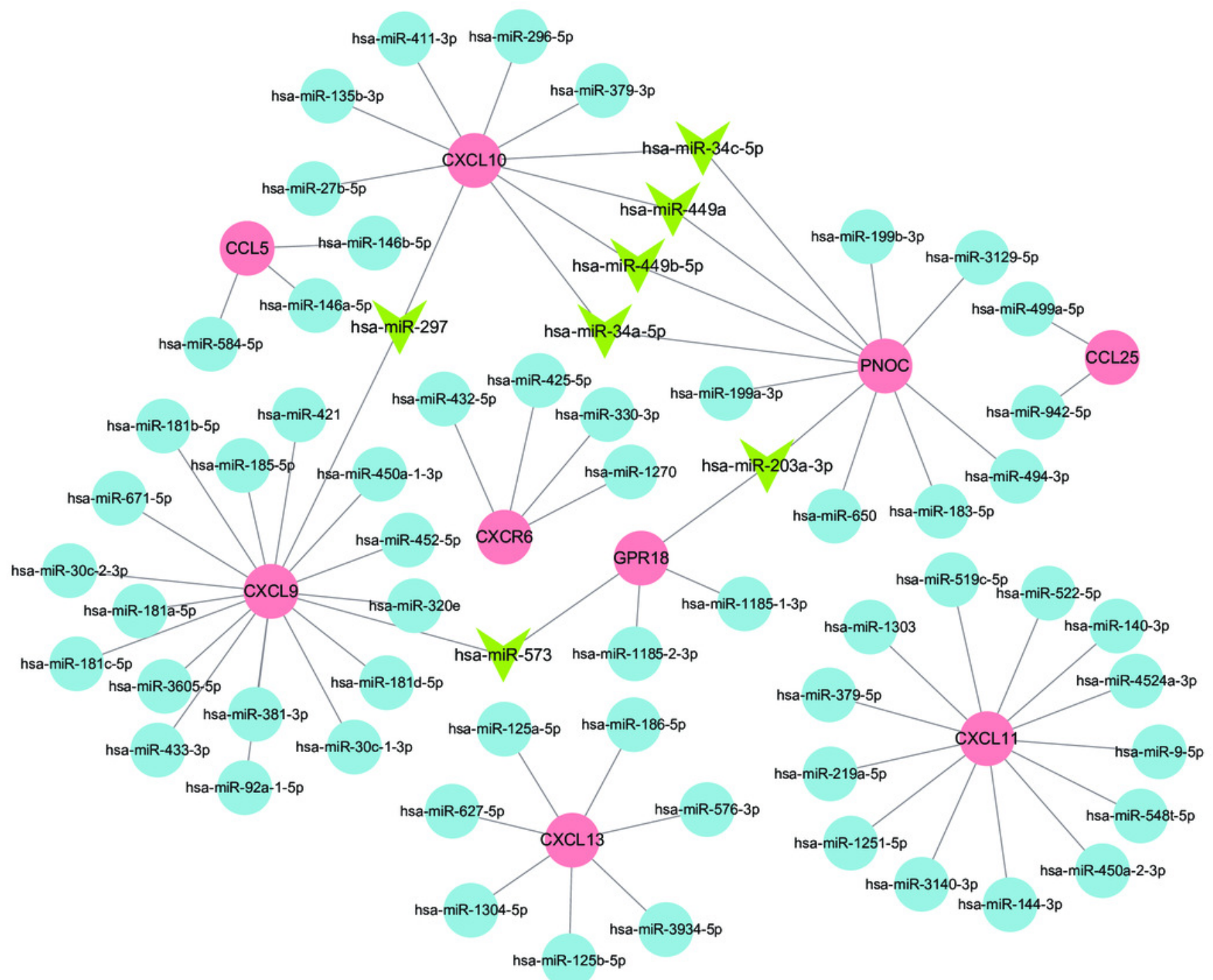


Figure 9

Validation of hub genes by ROC curve analysis.

AUC value of each plot was calculated. Genes with AUC values greater than $85 \%$ were considered as potential biomarkers for the diagnosis of RA. (A) CCL25, (B) CXCL10, (C) CCL5, (D) GPR18, (E) CXCL9, (F) PNOC, (G) CXCL13, (H) CXCL11, (I) CXCR6. 
A

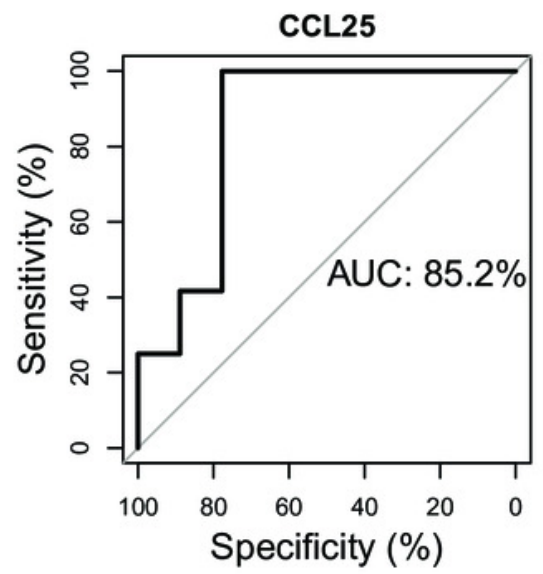

D

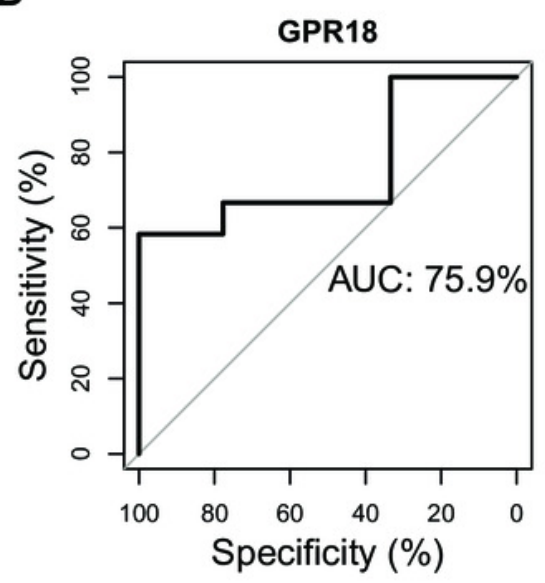

G

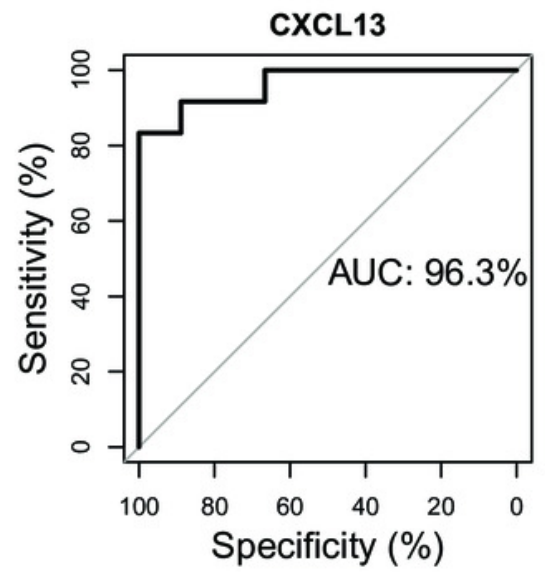

B

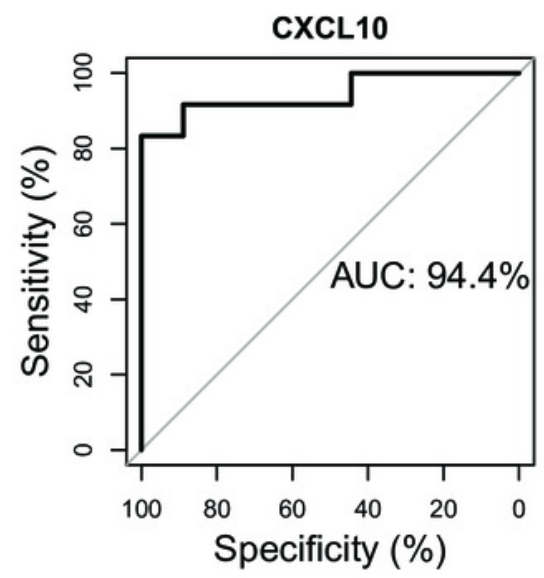

E

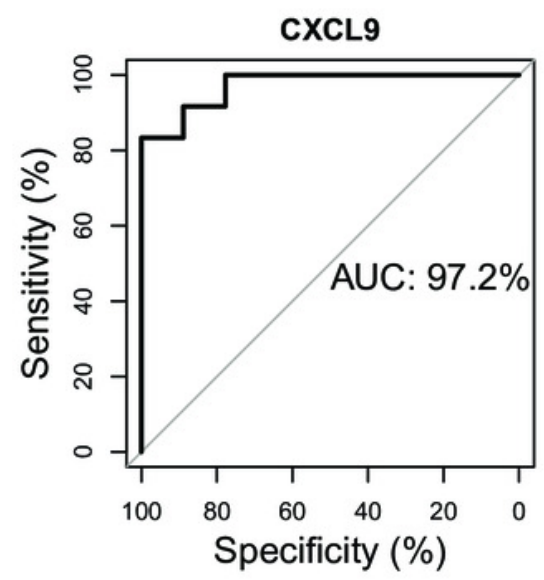

H

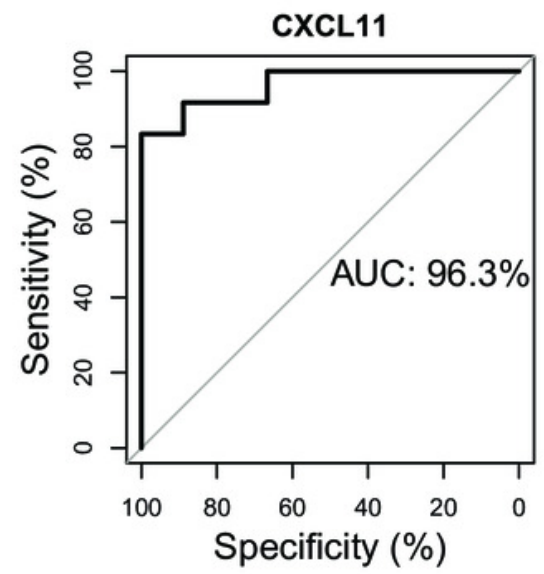

C

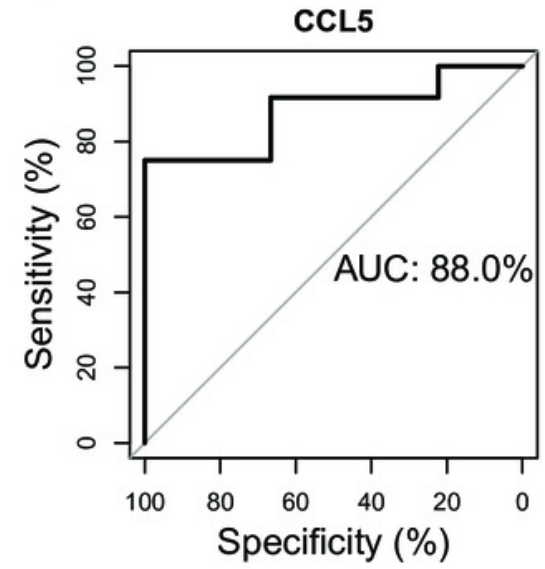

$\mathbf{F}$

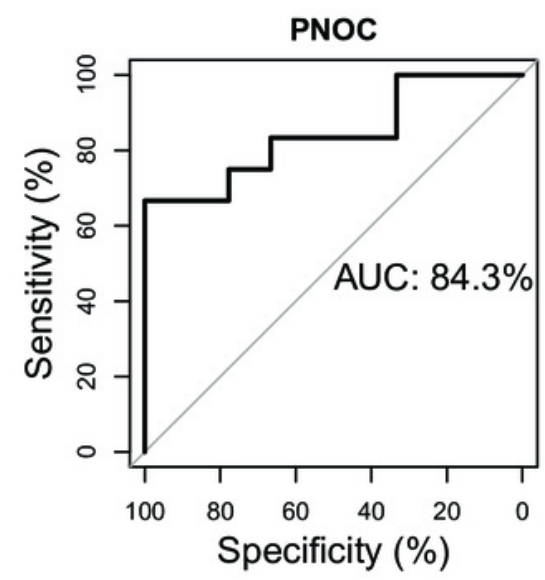

I

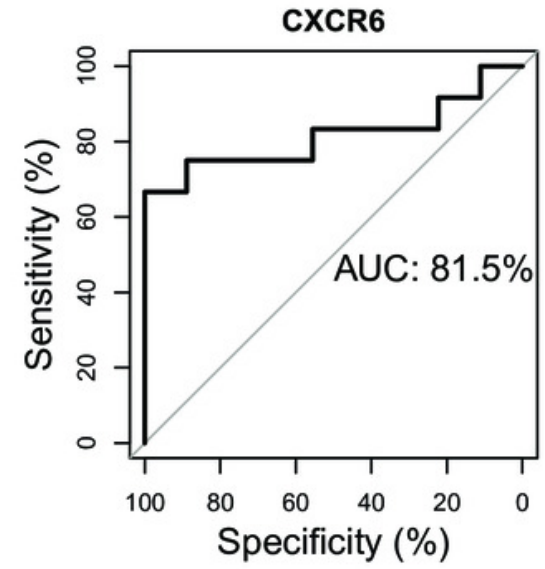


Figure 10

Validation of hub genes by RT-qPCR and Western blot.

(A) RT-qPCR validation of the hub gene between RA and normal controls. (B) Expression of

CXCL10 in PBMCs was detected with Western blot. (C) The relative expression of CXCL10 was quantified via normalization to $\beta$-actin. * $p$-value $<0.05$; ** p-value $<0.01$. 
A

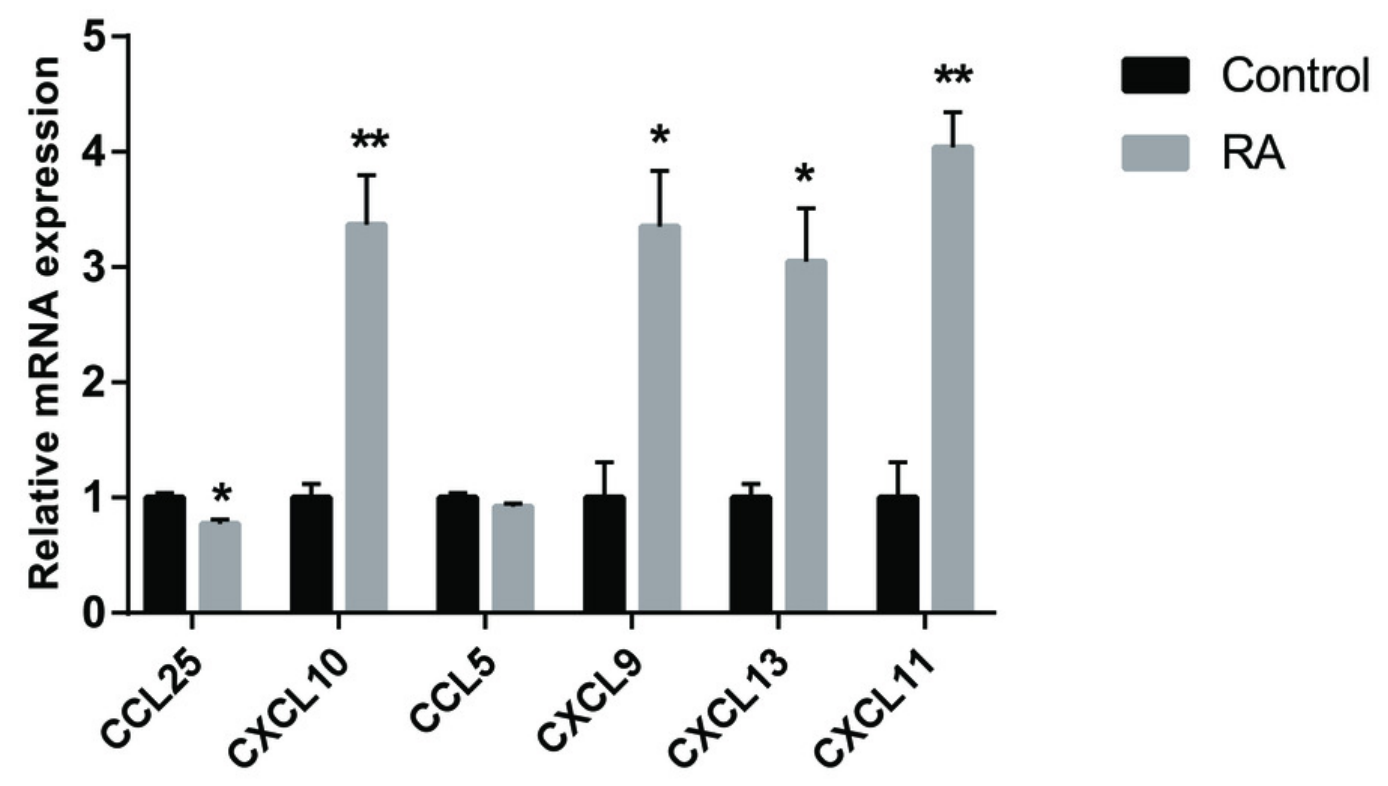

B

C

CXCL10

CXCL10

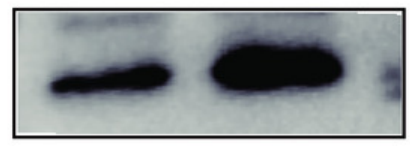

$\beta$-actin
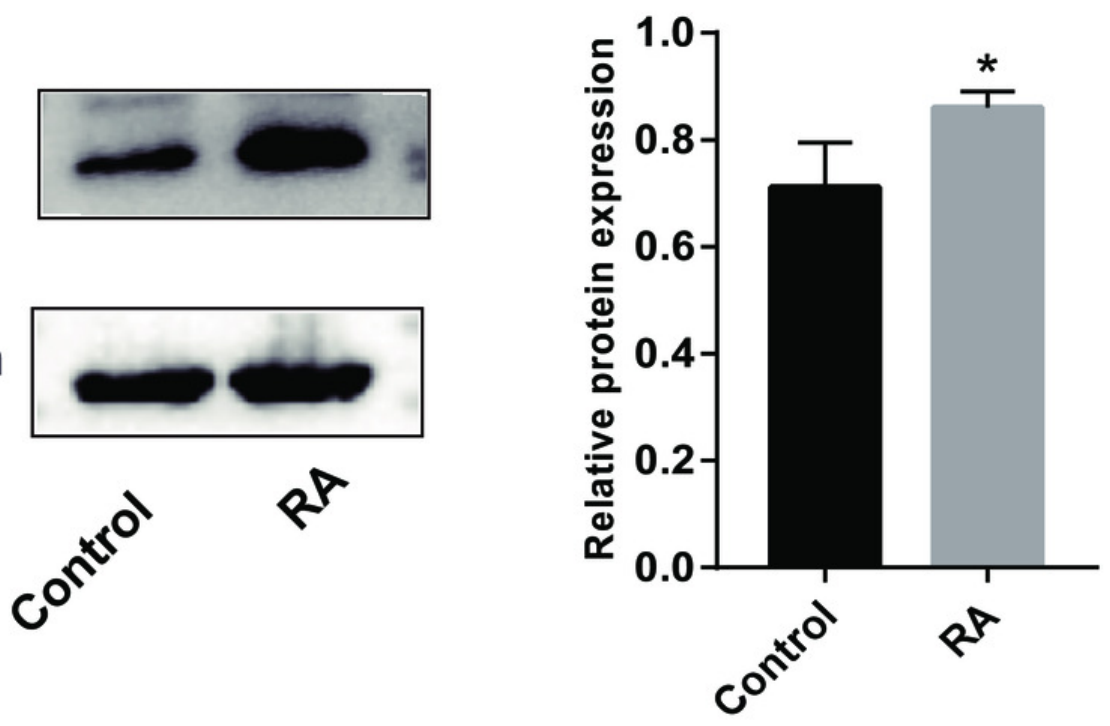
Figure 11

Validation of vital microRNAs.

(A-F) The relative expressions of hsa-miR-34c-5p, hsa-miR-449a, hsa-miR-449b, hsa-miR-34a, hsa-miR-573, and hsa-miR-203 in synovial tissue. Data were obtained from the GEO dataset GSE37425 and presented as log2 expression of probe intensity (health control: $n=2, R A$ patients: $n=4)$. (G) hsa-miR-34a-5p expression in PBMCs was detected with RT-qPCR. Data were relative to U6 expression. ${ }^{*} p$-value $<0.05,{ }^{* * *}$-value $<0.001$. 
A

hsa-miR-34c-5p

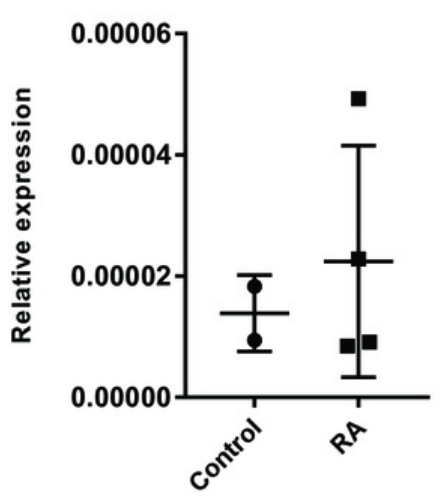

D

hsa-miR-34a

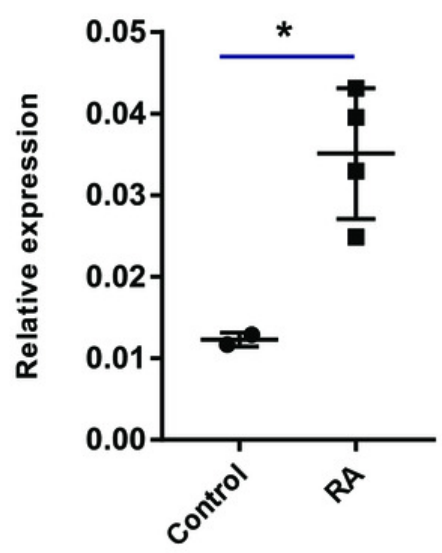

G

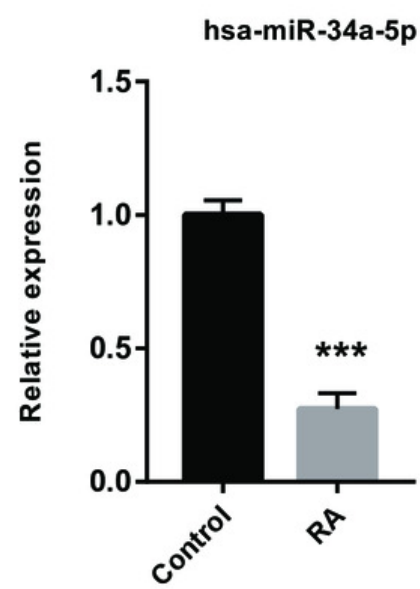

B

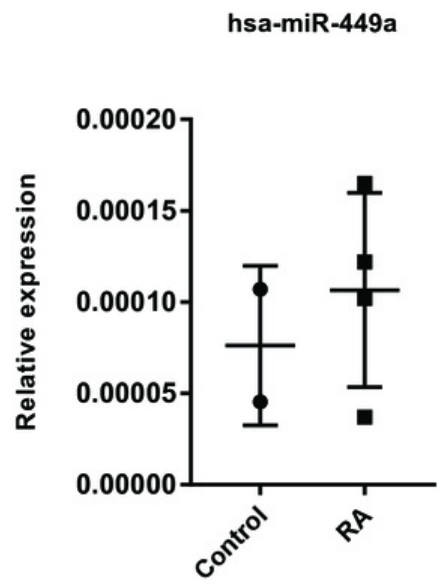

$\mathbf{E}$

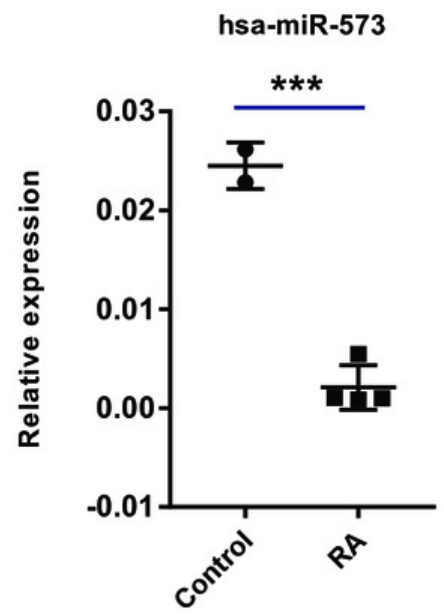

C

hsa-miR-449b

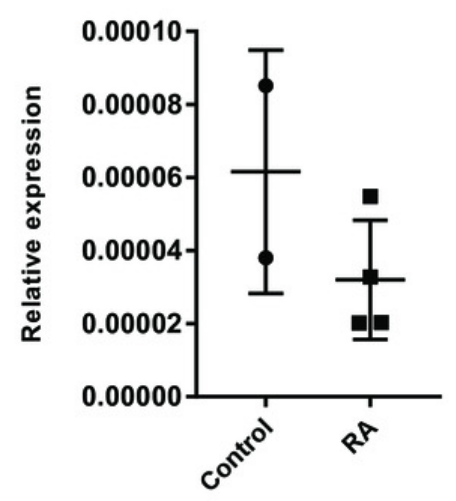

$\mathbf{F}$

hsa-miR-203

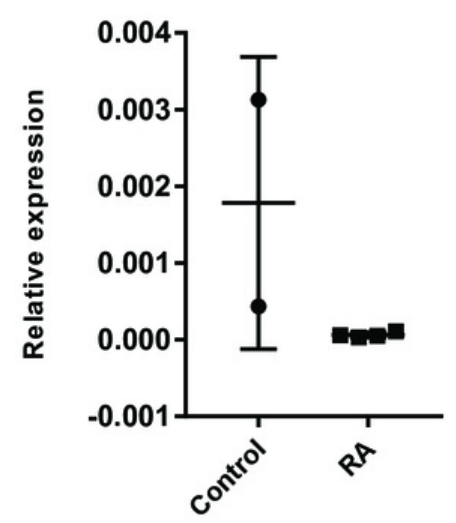




\section{Table 1 (on next page)}

RT-qPCR primers used in this study. 
1 Table 1 RT-qPCR primers used in this study

\begin{tabular}{cl}
\hline Gene symbol & \multicolumn{1}{c}{ Sequence $\left(5^{\prime}\right.$ to $\left.3^{\prime}\right)$} \\
\hline CCL25 & forward: TATTCTACCTCCCCAAGAGACA \\
& reverse: GATGGGATTGCTAAACTTGGAC \\
CXCL10 & forward: CTCTCTCTAGAACTGTACGCTG \\
& reverse: ATTCAGACATCTCTTCTCACCC \\
CCL5 & forward: CAGCAGTCGTCCACAGGTCAAG \\
& reverse: TTTCTTCTCTGGGTTGGCACACAC \\
CXCL9 & forward: AAGACCTTAAACAATTTGCCCC \\
& reverse: TGCTGAATCTGGGTTTAGACAT \\
CXCL13 & forward: CAAGGTGTTCTGGAGGTCTATT \\
& reverse: TGAATTCGATCAATGAAGCGTC \\
CXCL11 & forward: GCTGTGATATTGTGTGCTACAG \\
& reverse: TTGGGTACATTATGGAGGCTTT \\
GAPDH & forward: CGGACCAATACGACCAAATCCG \\
& reverse: AGCCACATCGCTCAGACACC \\
miR-34a-5p & forward: TCTGTCTCTCTTGGCAGTGTCTTA \\
& reverse: AATGGTTGTTCTCCACTCTCTCTC \\
& forward: CAGCACATATACTAAAATTGGAACG \\
U6 & reverse: ACGAATTTGCGTGTCATCC
\end{tabular}

2 
Table 2 (on next page)

Detailed information of each cluster 
1 Table 2 Detailed information of each cluster

\begin{tabular}{lllll}
\hline Cluster & Score & Nodes & Edges & Node IDs \\
\hline 1 & 9.000 & 9 & 36 & CCL25, CCL5, CXCL10, CXCL11, CXCL13, CXCL9, \\
& & & & CXCR6, GPR18, PNOC \\
2 & 6.286 & 8 & 22 & BLNK, CD19, CD72, CD79A, CD79B, IGLL5, ITK, PLCG2 \\
3 & 4.400 & 6 & 11 & CD8A, MAP4K1, CD247, TRAT1, CD3D, GZMA \\
4 & 4.000 & 4 & 6 & BIRC3, PSMB10, PSMB9, TNFSF11 \\
5 & 3.000 & 3 & 3 & CD2, CD48, CD52 \\
\hline
\end{tabular}

2 
Table 3 (on next page)

Vital miRNAs and its target genes 
1 Table 3 Vital miRNAs and its target genes

\begin{tabular}{lll}
\hline miRNA & Target genes & Count \\
\hline hsa-miR-297 & CXCL9, CXCL10 & 2 \\
hsa-miR-573 & CXCL9, GPR18 & 2 \\
hsa-miR-203a-3p & GPR18, PNOC & 2 \\
hsa-miR-449b-5p & PNOC, CXCL10 & 2 \\
hsa-miR-34c-5p & PNOC, CXCL10 & 2 \\
hsa-miR-449a & PNOC, CXCL10 & 2 \\
hsa-miR-34a-5p & PNOC, CXCL10 & 2 \\
\hline
\end{tabular}

2 